\title{
Photospheric flux density of magnetic helicity ${ }^{\star}$
}

\author{
E. Pariat ${ }^{1}$, P. Démoulin ${ }^{1}$, and M. A. Berger ${ }^{2}$ \\ 1 Observatoire de Paris, LESIA, UMR 8109 (CNRS), 92195 Meudon, France \\ e-mail: [etienne.pariat; pascal.demoulin]@obspm.fr \\ 2 Department of Mathematics, University College London, UK \\ e-mail: m.berger@ucl.ac.uk
}

Received 10 January 2005 / Accepted 12 May 2005

\begin{abstract}
Several recent studies have developed the measurement of magnetic helicity flux from the time evolution of photospheric magnetograms. The total flux is computed by summing the flux density over the analyzed region. All previous analyses used the density $G_{A}\left(=-2(\boldsymbol{A} \cdot \boldsymbol{u}) B_{n}\right)$ which involves the vector potential $\boldsymbol{A}$ of the magnetic field. In all the studied active regions, the density $G_{A}$ has strong polarities of both signs with comparable magnitude. Unfortunately, the density $G_{A}$ can exhibit spurious signals which do not provide a true helicity flux density. The main objective of this study is to resolve the above problem by defining the flux of magnetic helicity per unit surface. In a first step, we define a new density, $G_{\theta}$, which reduces the fake polarities by more than an order of magnitude in most cases (using the same photospheric data as $G_{A}$ ). In a second step, we show that the coronal linkage needs to be provided in order to define the true helicity flux density. It represents how all the elementary flux tubes move relatively to a given elementary flux tube, and the helicity flux density is defined per elementary flux tube. From this we define a helicity flux per unit surface, $G_{\Phi}$. We show that it is a field-weighted average of $G_{\theta}$ at both photospheric feet of coronal connections. We compare these three densities $\left(G_{A}, G_{\theta}, G_{\Phi}\right)$ using theoretical examples representing the main cases found in magnetograms (moving magnetic polarities, separating polarities, one polarity rotating around another one and emergence of a twisted flux tube). We conclude that $G_{\theta}$ is a much better proxy of the magnetic helicity flux density than $G_{A}$ because most fake polarities are removed. Indeed $G_{\theta}$ gives results close to $G_{\Phi}$ and should be used to monitor the photospheric injection of helicity (when coronal linkages are not well known). These results are applicable to the results of any method determining the photospheric velocities. They can provide separately the flux density coming from shearing and advection motions if plasma motions are known.
\end{abstract}

Key words. Sun: magnetic fields - Sun: photosphere - Sun: corona

\section{Introduction}

The theoretical importance of magnetic helicity in understanding the magnetohydrodynamic (MHD) evolution of magnetic fields was recognized a long time ago (e.g. Berger \& Field 1984). However it is only during the last years that attempts were made to measure magnetic helicity from solar and interplanetary observations. Magnetic helicity (unlike current helicity) is one of the few global quantities that is conserved even in resistive MHD on a time-scale less than the global diffusion time-scale. Thus magnetic helicity studies make it possible to trace helicity as it emerges from the sub-photospheric layers to the corona and then is ejected via coronal mass ejections (CMEs) into interplanetary space, reaching the Earth as magnetic clouds (MCs).

Presently most developments have been done at the photospheric level because the magnetic field is mainly measured there in the solar atmosphere. The flux of magnetic helicity from differential motion is usually found to be too small (typically a factor 10) to explain the helicity ejected in CMEs

* Appendix A is only available in electronic form at http://www. edpsciences.org
(Démoulin et al. 2002; Green et al. 2002; Nindos et al. 2003). Most of the helicity flux comes from local motions within ARs (e.g. Nindos et al. 2003; Chae et al. 2004; Kusano et al. 2005), with a negligible contribution from the quiet Sun (Welsch \& Longcope 2003).

The horizontal photospheric velocities, needed to compute the helicity flux, are determined by tracking the evolution of the magnetic field. The most widely used are local correlation tracking (LCT) methods. Two important parameters in LCT have to be properly chosen: the FWHM (full width at half maximum) of the apodizing function and the time interval between the two images to be compared. Chae et al. (2004) made a quantitative estimate of the effects of both parameters. They found that, when averaged over a time period of, or larger than, one hour the average flux of helicity is the same within $10 \%$. The LCT method still has limitations (Démoulin \& Berger 2003; Kusano et al. 2005), e.g. it gives only the mean velocity of several flux tubes (the average is done by the apodizing function which needs to be several pixels width). Then Kusano et al. (2002) proposed a method which uses the vertical component of the induction equation. In fact the velocity of flux tubes cannot fully be deduced from the induction equation and part 
of the velocity is still computed from the LCT method (Welsch et al. 2004). When the transverse magnetic field is available, Kusano et al. (2005) developed a method which minimize the input from the LCT, while Longcope (2004) proposed a method which uses only the induction equation and minimizes the photospheric "kinetic energy".

Whatever is the method used to derive the photospheric velocities, the injection of magnetic helicity has complicated patterns both in space and time. In particular, Chae et al. (2001, 2004); Kusano et al. (2002, 2005); Moon et al. (2002b); Nindos et al. (2003) found that the total flux of magnetic helicity over an AR is usually the summation of opposite sign contributions which usually have similar magnitude (then the net flux is much lower than the unsigned flux).

Why does the derived helicity flux have so much mixedsign polarity? It could come from the effect of turbulence on the magnetic flux tubes in the convection zone. But before looking for any physical implications, either in the convective zone, or in the corona, the notion of helicity flux (per unit surface) should be re-visited. Magnetic helicity is a global quantity, and it is not obvious if a helicity density and a helicity flux per unit surface have any physical meaning.

The definition of the relative magnetic helicity and its injection via boundary motions are briefly summarized in Sect. 2. Then we explore three different possibilities to define a helicity flux per unit surface: the first one, $G_{A}$, was used to analyze all previous observations (Sect. 3.1), a second one, $G_{\theta}$, which gives better results (Sect. 3.2) and a third one, $G_{\Phi}$, which has the required property of a density (Sect. 3.3). The two first densities, $G_{A}$ and $G_{\theta}$, can be computed with only photospheric observations while $G_{\Phi}$ needs the coronal connectivities which are, in practice, difficult to observe. We then explore, with basic configurations, the advantages of using $G_{\theta}$ rather than $G_{A}$, and compare with $G_{\Phi}$, in Sect. 4 . Then we conclude on the best way to derive the photospheric flux of magnetic helicity with present observations (Sect. 5).

\section{Magnetic helicity}

\subsection{Relative magnetic helicity}

The magnetic helicity of a divergence-free field $\boldsymbol{B}$ within a volume $\mathcal{V}$, bounded by a surface $\mathcal{S}$, was first defined by:

$H_{\mathrm{c}}=\int_{\mathcal{V}} \boldsymbol{A} \cdot \boldsymbol{B} \mathrm{d}^{3} x$

where the vector potential $\boldsymbol{A}$ satisfies $\boldsymbol{B}=\boldsymbol{\nabla} \times \boldsymbol{A}$. However Eq. (1) is gauge invariant (independent of $\Phi$ where $\boldsymbol{A}^{\prime}=\boldsymbol{A}+$ $\boldsymbol{\nabla} \Phi)$ only when the magnetic field is fully contained inside the volume $\mathcal{V}$ (i.e. at any point of $\mathcal{S}$, the normal component $B_{n}=$ $\boldsymbol{B} \cdot \hat{\boldsymbol{n}}$ vanishes).

In the case where $\mathcal{V}$ is part of the corona, we clearly have magnetic fluxes crossing $\mathcal{S}$ (in particular for the part of $\mathcal{S}$ located at the photospheric level). Berger \& Field (1984) have shown that for cases where $B_{n} \neq 0$ on $\mathcal{S}$ one can define a relative magnetic helicity, $H$, by subtracting the helicity of the potential field $\boldsymbol{B}_{\mathrm{p}}\left(\boldsymbol{B}_{\mathrm{p}}\right.$ has the same normal component $B_{n}$ on $\left.\mathcal{S}\right)$.
An expression for $H$, valid for any gauge is (Finn \& Antonsen 1985):

$H=\int_{\mathcal{V}}\left(\boldsymbol{A}+\boldsymbol{A}_{\mathrm{p}}\right) \cdot\left(\boldsymbol{B}-\boldsymbol{B}_{\mathrm{p}}\right) \mathrm{d}^{3} x$.

\subsection{Flux of magnetic helicity}

The time variation of $H$ can be written in general as (Berger \& Field 1984):

$$
\begin{aligned}
\left.\frac{\mathrm{d} H}{\mathrm{~d} t}\right|_{\mathrm{Tot}}= & 2 \int_{\mathcal{S}}\left(\boldsymbol{A}_{\mathrm{p}} \times \boldsymbol{E}\right) \mathbf{d} \boldsymbol{S} \\
& -2 \int_{\mathcal{V}} \boldsymbol{E} \cdot \boldsymbol{B} \mathrm{d}^{3} x-2 \int_{\mathcal{S}} \frac{\partial \Psi}{\partial t} \boldsymbol{A}_{\mathrm{p}} \cdot \mathbf{d} \mathcal{S},
\end{aligned}
$$

where $\Psi$ is defined by $\boldsymbol{B}_{\mathrm{p}}=\boldsymbol{\nabla} \Psi$ (potential field), $\boldsymbol{E}$ is the electric field associated to $\boldsymbol{B}$ evolution, and $\mathbf{d} \boldsymbol{S}$ is the elementary surface directed outside the volume $\mathcal{V}$. The last term on the right has been computed with the gauge:

$\boldsymbol{\nabla} \cdot \boldsymbol{A}_{\mathrm{p}}=0$.

Below we will always use this gauge. The last term of Eq. (3) vanishes if we select the boundary condition:

$\boldsymbol{A}_{\mathrm{p}} \cdot \hat{\boldsymbol{n}}=0$,

with $\hat{\boldsymbol{n}} \mathrm{d} \mathcal{S}=-\mathbf{d} \mathcal{S}$. This choice is compatible with the gauge selection (Eq. (4)).

With $\boldsymbol{E}=-\boldsymbol{v} \times \boldsymbol{B}+\boldsymbol{R}$ in resistive MHD, $\boldsymbol{R}$ being the nonideal resistive term, Eq. (3) becomes:

$$
\begin{aligned}
\left.\frac{\mathrm{d} H}{\mathrm{~d} t}\right|_{\mathrm{Tot}}= & 2 \int_{\mathcal{S}}\left[\left(\boldsymbol{A}_{\mathrm{p}} \cdot \boldsymbol{B}\right) v_{n}-\left(\boldsymbol{A}_{\mathrm{p}} \cdot \boldsymbol{v}\right) B_{n}\right] \mathrm{d} \mathcal{S} \\
& -2 \int_{\mathcal{S}}\left(\boldsymbol{A}_{\mathrm{p}} \times \boldsymbol{R}\right)_{n} \mathrm{~d} \mathcal{S}-2 \int_{\mathcal{V}} \boldsymbol{R} \cdot \boldsymbol{B} \mathrm{d}^{3} x .
\end{aligned}
$$

Since the dissipation of magnetic helicity is very small (Berger 1984), we consider below only the first integral, i.e. the ideal MHD flux of helicity. With the boundary condition given by Eq. (5), the two terms of the integral have a clear physical interpretation: the first term corresponds to inflow (or outflow) of helicity through $\mathcal{S}$ (advection term), while the second term corresponds to helicity flux by footpoint motions parallel to $\mathcal{S}$ (shear term).

\subsection{Estimation of the flux from observations}

Present magnetograms provide $\boldsymbol{B}$ on $\mathcal{S}$ located in the photosphere. From $B_{n}$ distribution on $\mathcal{S}$ and Eq. (4) one can derive the vector potential $\boldsymbol{A}_{\mathrm{p}}$ (an explicit formula is given below in Eq. (14)). The observed evolution of the magnetic field can provide an estimation of the velocities. It is then possible to estimate the photospheric flux of helicity from present observations.

Initially, since only horizontal velocities were deduced from the temporal evolution of $B_{n}$, it was supposed that only the shear term could be derived (e.g. Chae et al. 2001; Nindos \& Zhang 2002; Moon et al. 2002b). However, the magnetograms of $B_{n}$ permit us to follow the photospheric intersection of the 
magnetic flux-tubes but not the evolution of the plasma (even if the plasma is frozen in the field, the two velocities are usually different). From the observed magnetic evolution one can estimate the flux-tube motion, not the plasma motion, parallel to $\mathcal{S}$. Let $\boldsymbol{v}_{\mathrm{t}}$ be the plasma velocity tangent to the photosphere and $v_{n}$ be the velocity perpendicular to the photosphere. With the velocity of the footpoints of flux tubes, $\boldsymbol{u}$, defined as

$\boldsymbol{u}=\boldsymbol{v}_{\mathrm{t}}-\frac{v_{n}}{B_{n}} \boldsymbol{B}_{\mathrm{t}}$,

the helicity flux given by Eq. (6) simplifies to

$\frac{\mathrm{d} H}{\mathrm{~d} t}=-2 \int_{\mathcal{S}}\left(\boldsymbol{A}_{\mathrm{p}} \cdot \boldsymbol{u}\right) B_{n} \mathrm{~d} \mathcal{S}$,

allowing the full helicity flux to be deduced (Démoulin \& Berger 2003, but with several possible caveats as discussed therein). Nindos et al. (2003) have used Eq. (7) in order to derive the full helicity flux from observations.

Several methods have been developed to derive the velocities from observed $B_{n}$ evolution (see Sect. 1). The LCT method follow explicitly the magnetic flux, so derive an estimation of $\boldsymbol{u}$. But even the methods using the normal component of the ideal induction equation (combined in different ways with the LCT method) can only give an estimation of $\boldsymbol{u}$, since the induction equation can be rewritten as (Démoulin \& Berger 2003):

$\frac{\partial B_{n}}{\partial t}=-\nabla_{\mathrm{t}}\left(B_{n} \boldsymbol{u}\right)$.

Since flows along $\boldsymbol{B}$ have no effect on the helicity flux (they do not change $\boldsymbol{E}=-\boldsymbol{v} \times \boldsymbol{B}$ ), vector magnetograms allow us only to compute from $\boldsymbol{u}$ the velocity $\boldsymbol{v}_{\perp}$ locally orthogonal to $\boldsymbol{B}$ (Welsch et al. 2004). However, we cannot interpret the normal component $v_{\perp, n}$ as a plasma velocity crossing $\mathcal{S}$ (like for $v_{n}$ ). Since $v_{\perp, n}$ can be written as:

$v_{\perp, n}=\boldsymbol{v} \cdot \hat{\boldsymbol{n}}-\frac{(\boldsymbol{v} \cdot \boldsymbol{B})}{\boldsymbol{B}^{2}} \boldsymbol{B} \cdot \hat{\boldsymbol{n}}$

for example if we consider plasma motions only tangential to $\mathcal{S}$, then $v_{\perp, n} \neq 0$ at every location where $\boldsymbol{B}$ is not orthogonal to $\boldsymbol{v}$. This leads to the false impression that some flows are crossing $\mathcal{S}$.

The above arguments imply that we cannot fully separate the shear and advection terms in Eq. (6) if we are using the LCT or/and the induction equation to derive the velocities. The only part which can be separated un-ambiguously is the part of the shearing term associated to the velocity component orthogonal both to $\hat{\boldsymbol{n}}$ and $\boldsymbol{B}_{\mathrm{t}}$ (this part cannot come from emergence as shown by Eq. (7)). The other part of the shearing term can always be re-interpreted as an advection term and vice versa.

In order to separate the shearing and advection terms, we need to derive the full plasma velocity vector $\boldsymbol{v}$. Measuring the Doppler velocity gives only one component of the plasma velocity but it can be combined with the determination of $\boldsymbol{u}$ to get an estimate of $\boldsymbol{v}$. The method of Longcope (2004) provides this possibility within the framework of minimizing a functional. Then, if we know both $\boldsymbol{v}$ and $\boldsymbol{B}$, we can separate the shearing and advection terms in Eq. (6). However we can always rewrite them as in Eq. (8) with $\boldsymbol{u}$ replaced by $\boldsymbol{u}_{\text {shear }}=\boldsymbol{v}_{\mathrm{t}}$ for the shearing term, and $\boldsymbol{u}_{\text {advec }}=-\boldsymbol{B}_{\mathrm{t}} v_{n} / \boldsymbol{B}_{n}$ for the advection term. So we have:

$$
\begin{aligned}
& \left.\frac{\mathrm{d} H}{\mathrm{~d} t}\right|_{\text {shear }}=-2 \int_{\mathcal{S}}\left(\boldsymbol{A}_{\mathrm{p}} \cdot \boldsymbol{v}_{\mathrm{t}}\right) B_{n} \mathrm{~d} \mathcal{S}, \\
& \left.\frac{\mathrm{d} H}{\mathrm{~d} t}\right|_{\text {advec }}=-2 \int_{\mathcal{S}}\left(\boldsymbol{A}_{\mathrm{p}} \cdot \boldsymbol{u}_{\text {advec }}\right) B_{n} \mathrm{~d} \mathcal{S} .
\end{aligned}
$$

Below we use $\boldsymbol{u}$ generically but all the results apply to shear and advection terms separately or to their sum depending on the data available.

\section{Flux density of magnetic helicity}

\subsection{Flux density involving the vector potential}

Equations (8), (11), (12) seem to suggest that the quantity

$G_{A}(\boldsymbol{x})=-2\left(\boldsymbol{A}_{\mathrm{p}} \cdot \boldsymbol{u}\right) B_{n}$

could be a candidate for the helicity flux density. $G_{A}$ has been used extensively to monitor the spatial distribution of magnetic helicity flux in previous studies of active regions (Chae 2001; Chae et al. 2001, 2004; Kusano et al. 2002, 2005; Nindos \& Zhang 2002; Nindos et al. 2003; Moon et al. 2002a,b).

However $G_{A}$ is non null even with simple flows which do not input any magnetic helicity to the coronal field! For example, consider a simple circular magnetic region (with $B_{n}>0$ ) moving in a straight line. In the classical Coulomb gauge, the vector field $\boldsymbol{A}_{\mathrm{p}}$ is toroidal, while $\boldsymbol{u}$ is constant (say, $\boldsymbol{u}=U_{0} \hat{\boldsymbol{e}}_{x}$ ). Then $G_{A}$ will be positive in the top half $(y>0)$ of the region, and negative in the bottom half (see Fig. 1). Indeed $G_{A}$ introduces artificial polarities of both signs with most of the flow patterns. These amplitudes are analyzed for some basic flows in Sect. 4.

\subsection{Flux density involving relative velocities}

In order to solve the above problem, $\boldsymbol{A}_{\mathrm{p}}$ should be explicitly written in terms of the magnetic field (using the gauge of Eq. (4)). For simplicity we assume that the solar photosphere $\mathcal{S}$ is planar. In this geometry the field $\boldsymbol{A}_{\mathrm{p}}$ is especially simple (Berger 1984):

$A_{\mathrm{p}}(\boldsymbol{x})=\frac{1}{2 \pi} \hat{\boldsymbol{n}} \times \int_{\mathcal{S}^{\prime}} B_{n}\left(\boldsymbol{x}^{\prime}\right) \frac{\boldsymbol{r}}{r^{2}} \mathrm{~d} \mathcal{S}^{\prime}$,

where $\boldsymbol{r}=\boldsymbol{x}-\boldsymbol{x}^{\prime}$ is the vector between the two photospheric positions defined by $\boldsymbol{x}$ and $\boldsymbol{x}^{\prime}$.

Using Eq. (14), we obtain an expression for $\mathrm{d} H / \mathrm{d} t$ (Eq. (8), but also Eqs. (11), (12)) that depends only on observable photospheric quantities $\left(B_{n}, \boldsymbol{r}\right.$ and $\left.\boldsymbol{u}\right)$ :

$\frac{\mathrm{d} H}{\mathrm{~d} t}=-\left.\frac{1}{\pi} \int_{\mathcal{S}} \int_{\mathcal{S}^{\prime}} \frac{\boldsymbol{r} \times \boldsymbol{u}(\boldsymbol{x})}{r^{2}}\right|_{n} B_{n}(\boldsymbol{x}) B_{n}\left(\boldsymbol{x}^{\prime}\right) \mathrm{d} \mathcal{S} \mathrm{d} \mathcal{S}^{\prime}$.

Below we will let $B_{n}=B_{n}(\boldsymbol{x}), B_{n}^{\prime}=B_{n}\left(\boldsymbol{x}^{\prime}\right)$, etc. Since both integrations are done on the same surfaces, $\mathcal{S}=\mathcal{S}^{\prime}$, we can exchange $\boldsymbol{x}$ and $\boldsymbol{x}^{\prime}$. This yields a new equation that summed up with Eq. (15) gives:

$2 \frac{\mathrm{d} H}{\mathrm{~d} t}=-\left.\frac{1}{\pi} \int_{\mathcal{S}} \int_{\mathcal{S}^{\prime}} \frac{\boldsymbol{r} \times\left[\boldsymbol{u}-\boldsymbol{u}^{\prime}\right]}{r^{2}}\right|_{n} B_{n} B_{n}^{\prime} \mathrm{d} \mathcal{S} \mathrm{d} \mathcal{S}^{\prime}$ 


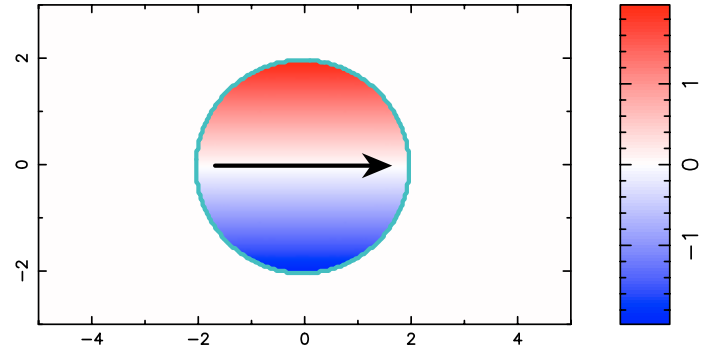

Fig. 1. Map of $G_{A}$ (Eq. (13)) for a single magnetic region executing a simple translational motion towards the right (arrow). The normal magnetic field component $B_{n}$ is uniform. The grey levels shows the strength of $G_{A}$ with middle grey being 0 , lighter grey positive, and darker grey negative (a color version is available in the electronic version at http://www . edpsciences . org with red/blue coding the positive/negative values). The computation has been done with $U_{0} R B_{0}^{2}=2$ (Eq. (30)).

Let us define $\theta$ as the angle between $\boldsymbol{r}$ and a fixed direction on $\mathcal{S}$ with trigonometric convention (counterclockwise), then

$$
\frac{\mathrm{d} \theta(\boldsymbol{r})}{\mathrm{d} t}=\frac{1}{r^{2}}\left(\boldsymbol{r} \times \frac{\mathrm{d} \boldsymbol{r}}{\mathrm{d} t}\right)_{n}=\frac{1}{r^{2}}\left(\boldsymbol{r} \times\left(\boldsymbol{u}-\boldsymbol{u}^{\prime}\right)\right)_{n} .
$$

Thus $\mathrm{d} \theta / \mathrm{d} t$ is the relative rotation rate of the two points $\boldsymbol{x}$ and $\boldsymbol{x}^{\prime}$. This rate is independent of the choice of the fixed direction used to define $\theta$. It is an intrinsic quantity of the motion pattern.

Equation (16) is transformed to

$$
\frac{\mathrm{d} H}{\mathrm{~d} t}=-\frac{1}{2 \pi} \int_{\mathcal{S}} \int_{\mathcal{S}^{\prime}} \frac{\mathrm{d} \theta(\boldsymbol{r})}{\mathrm{d} t} B_{n} B_{n}^{\prime} \mathrm{d} \mathcal{S} \mathrm{d} \mathcal{S}^{\prime} .
$$

This equation shows that the helicity injection rate can be understood as the summation of the rotation rate of all the pairs of elementary fluxes weighted with their magnetic flux (Berger 1986). It could then appear logical to define a helicity flux density $G_{\theta}(\boldsymbol{x})$ as:

$$
G_{\theta}(\boldsymbol{x})=-\frac{B_{n}}{2 \pi} \int_{\mathcal{S}^{\prime}} \frac{\mathrm{d} \theta(\boldsymbol{r})}{\mathrm{d} t} B_{n}^{\prime} \mathrm{d} \mathcal{S}^{\prime}
$$

This flux density is related to the previous definition, $G_{A}$ (Eq. (13)), as follow.

$$
\begin{aligned}
G_{\theta}= & \frac{B_{n}}{2 \pi} \hat{\boldsymbol{n}} \cdot \boldsymbol{u} \times \int_{\mathcal{S}^{\prime}} \frac{\boldsymbol{r}}{r^{2}} B_{n}^{\prime} \mathrm{d} \mathcal{S}^{\prime} \\
& -\frac{B_{n}}{2 \pi} \int_{\mathcal{S}^{\prime}}\left(\frac{\boldsymbol{u}^{\prime} \times \boldsymbol{r}}{r^{2}}\right)_{n} B_{n}^{\prime} \mathrm{d} \mathcal{S}^{\prime} \\
= & \frac{G_{A}}{2}-\frac{B_{n}}{2 \pi} \int_{\mathcal{S}^{\prime}}\left(\frac{\boldsymbol{u}^{\prime} \times \boldsymbol{r}}{r^{2}}\right)_{n} B_{n}^{\prime} \mathrm{d} \mathcal{S}^{\prime} .
\end{aligned}
$$

The first term $\left(G_{A} / 2\right)$ represents the motion of the endpoint at $\boldsymbol{x}$ around the rest of the photospheric magnetic field. But this gives only part of the net rotation of $\boldsymbol{x}$ about the field. To obtain all of $\mathrm{d} \theta / \mathrm{d} t$ we need the second term, which gives the motion of the rest of the field around the point $\boldsymbol{x}$. This is why $G_{A}$ by itself can produce spurious signals: it fails to accurately measure the net rotation of $\boldsymbol{x}$ about the photospheric field.

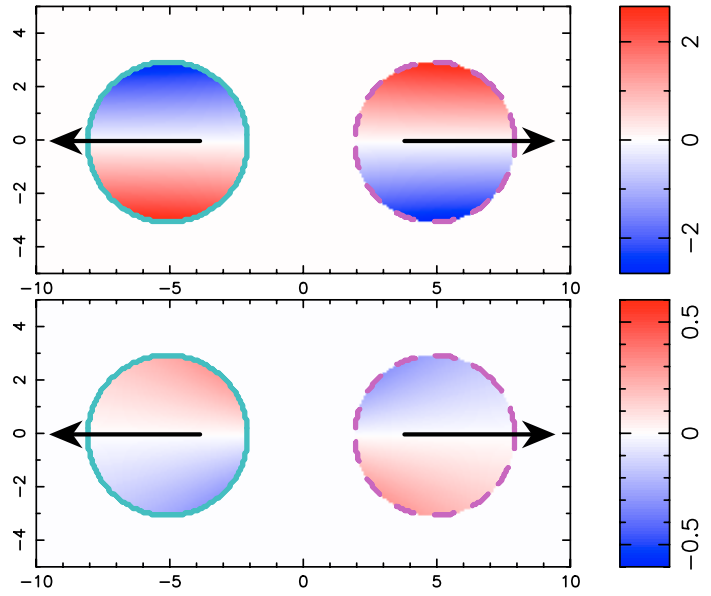

Fig. 2. Maps of $G_{A}$ and $G_{\theta}$ (Eqs. (13) and (19)), top and bottom panel respectively, for two magnetic regions of opposite polarity executing a simple translational motion away from each other (as indicated by arrows). $B_{n}$ is uniform in both magnetic polarities. Both $G_{A}$ and $G_{\theta}$ have two polarities in each magnetic region but with opposite sign and different magnitude $\left(G_{\theta}\right.$ is lower by about a factor 10 , see Fig. 4$)$. The shading convention is the same than in Fig. 1 (a color version is available in the electronic version at http://www.edpsciences.org). The values used are: $U_{0} R B_{0}^{2}=3$ and $D / R=10 / 3$.

For the non-rotating motion of a single footpoint illustrated in Fig. 1, we have $G_{\theta}=0$ everywhere. Thus $G_{\theta}$ does not suffer the same problem as $G_{A}$, which displays two artificial polarities.

However, we can still find configurations where $G_{\theta}$ does not give physically sensible results. For example, consider the case when two magnetic regions simply separate without any rotation. The total helicity flux is zero. However, as seen from a position $\boldsymbol{x}_{1}$ within region 1 , some points in region 2 will change their relative angle; i.e. $\mathrm{d} \theta\left(\boldsymbol{x}-\boldsymbol{x}^{\prime}\right) / \mathrm{d} t \neq 0$. For positions off axis, there will be a net contribution to $G_{\theta}$ (Fig. 2).

\subsection{Flux density involving field-line connectivities}

The definition of $G_{\theta}$ (Eq. (19)) has not fully reached our goal of defining a flux density of magnetic helicity. For that purpose we need to consider the magnetic connectivity in the volume $\mathcal{V}$ as shown below.

The magnetic helicity injected (Eq. (18)) can be separated into two terms:

$$
\begin{aligned}
\frac{\mathrm{d} H}{\mathrm{~d} t}= & +\frac{1}{2 \pi} \iint_{B_{n} \cdot B_{n}^{\prime}<0} \frac{\mathrm{d} \theta}{\mathrm{d} t}\left|B_{n} B_{n}^{\prime}\right| \mathrm{d} \mathcal{S} \mathrm{d} \mathcal{S}^{\prime} \\
& -\frac{1}{2 \pi} \iint_{B_{n} \cdot B_{n}^{\prime}>0} \frac{\mathrm{d} \theta}{\mathrm{d} t} B_{n} B_{n}^{\prime} \mathrm{d} \mathcal{S} \mathrm{d} \mathcal{S}^{\prime} .
\end{aligned}
$$

The first term, with $B_{n} \cdot B_{n}^{\prime}<0$, corresponds to the relative rotation of positive and negative polarities. For a configuration where a flux tube emerging into the corona from the convection zone links the opposite polarities, this term reflects the global shape, more precisely the writhe, of the flux tube. The last term corresponds to the flux of helicity by the rotation of each polarity $\left(B_{n} \cdot B_{n}^{\prime}>0\right)$. This helicity is injected in a similar way as twisting motions would do. 


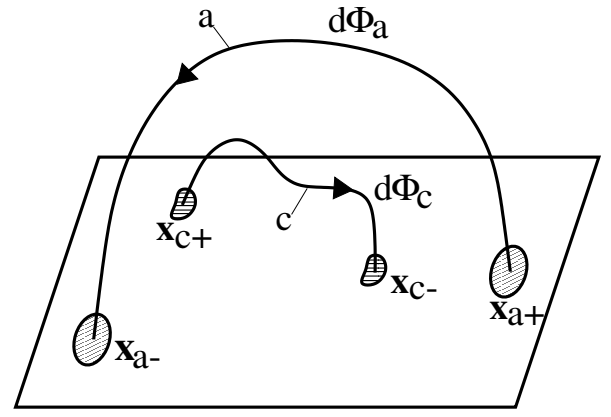

Fig. 3. Sketch of the general connectivity of two field lines, $a$ and $c$. The field line $a$ stretches from $\boldsymbol{x}_{a_{+}}$, where the magnetic field flux is $\mathrm{d} \Phi_{a_{+}}$, to $\boldsymbol{x}_{a_{-}}$with flux $\mathrm{d} \Phi_{a_{-}}$. Similarly, the footpoint positions of the $c$ field line, $\boldsymbol{x}_{c_{+}}$and $\boldsymbol{x}_{c_{-}}$, are respectively associated with a positive flux $\mathrm{d} \Phi_{c_{+}}$and a negative one $\mathrm{d} \Phi_{c_{-}}$. Conservation of the magnetic flux along the flux tubes gives: $\mathrm{d} \Phi_{a}=\mathrm{d} \Phi_{a_{+}}=\mathrm{d} \Phi_{a_{-}}$and $\mathrm{d} \Phi_{c}=$ $\mathrm{d} \Phi_{c_{+}}=\mathrm{d} \Phi_{c_{-}}$.

Let us define the elementary fluxes: in the positive polarity $\mathrm{d} \Phi_{+}=B_{n}\left(\boldsymbol{x}_{+}\right) \mathrm{d} \mathcal{S}$ and a similar one for the negative polarity: $\mathrm{d} \Phi_{-}=-B_{n}\left(\boldsymbol{x}_{-}\right) \mathrm{d} \mathcal{S}$. Then we get:

$$
\begin{aligned}
\frac{\mathrm{d} H}{\mathrm{~d} t}= & +\frac{1}{2 \pi} \int_{\Phi_{-}} \int_{\Phi_{+}^{\prime}} \frac{\mathrm{d} \theta\left(\boldsymbol{x}_{-}-\boldsymbol{x}_{+}^{\prime}\right)}{\mathrm{d} t} \mathrm{~d} \Phi_{-} \mathrm{d} \Phi_{+}^{\prime} \\
& +\frac{1}{2 \pi} \int_{\Phi_{+}} \int_{\Phi_{-}^{\prime}} \frac{\mathrm{d} \theta\left(\boldsymbol{x}_{+}-\boldsymbol{x}_{-}^{\prime}\right)}{\mathrm{d} t} \mathrm{~d} \Phi_{+} \mathrm{d} \Phi_{-}^{\prime} \\
& -\frac{1}{2 \pi} \int_{\Phi_{+}} \int_{\Phi_{+}^{\prime}} \frac{\mathrm{d} \theta\left(\boldsymbol{x}_{+}-\boldsymbol{x}_{+}^{\prime}\right)}{\mathrm{d} t} \mathrm{~d} \Phi_{+} \mathrm{d} \Phi_{+}^{\prime} \\
& -\frac{1}{2 \pi} \int_{\Phi_{-}} \int_{\Phi_{-}^{\prime}} \frac{\mathrm{d} \theta\left(\boldsymbol{x}_{-}-\boldsymbol{x}_{-}^{\prime}\right)}{\mathrm{d} t} \mathrm{~d} \Phi_{-} \mathrm{d} \Phi_{-}^{\prime} .
\end{aligned}
$$

In the above equation, the integrals are done at the boundary $\mathcal{S}$ without taking into account the existing connectivity in the volume $\mathcal{V}$.

Now let us organize the integrals of Eq. (22) according to the field line connectivity. Let $a$ be the label of a generic field line going from $\boldsymbol{x}_{a_{+}}$to $\boldsymbol{x}_{a_{-}}$Similarly, the field line labeled $c$ travels from $\boldsymbol{x}_{c_{+}}$to $\boldsymbol{x}_{c_{-}}$(Fig. 3). Then we can reorganize Eq. (22) as:

$$
\begin{aligned}
\frac{\mathrm{d} H}{\mathrm{~d} t}= & +\frac{1}{2 \pi} \int_{\Phi_{a_{-}}} \int_{\Phi_{c_{+}}} \frac{\mathrm{d} \theta\left(\boldsymbol{x}_{a_{-}}-\boldsymbol{x}_{c_{+}}\right)}{\mathrm{d} t} \mathrm{~d} \Phi_{a_{-}} \mathrm{d} \Phi_{c_{+}} \\
& +\frac{1}{2 \pi} \int_{\Phi_{a_{+}}} \int_{\Phi_{c_{-}}} \frac{\mathrm{d} \theta\left(\boldsymbol{x}_{a_{+}}-\boldsymbol{x}_{c_{-}}\right)}{\mathrm{d} t} \mathrm{~d} \Phi_{a_{+}} \mathrm{d} \Phi_{c_{-}} \\
& -\frac{1}{2 \pi} \int_{\Phi_{a_{+}}} \int_{\Phi_{c_{+}}} \frac{\mathrm{d} \theta\left(\boldsymbol{x}_{a_{+}}-\boldsymbol{x}_{c_{+}}\right)}{\mathrm{d} t} \mathrm{~d} \Phi_{a_{+}} \mathrm{d} \Phi_{c_{+}} \\
& -\frac{1}{2 \pi} \int_{\Phi_{a_{-}}} \int_{\Phi_{c_{-}}} \frac{\mathrm{d} \theta\left(\boldsymbol{x}_{a_{-}}-\boldsymbol{x}_{c_{-}}\right)}{\mathrm{d} t} \mathrm{~d} \Phi_{a_{-}} \mathrm{d} \Phi_{c_{-}} .
\end{aligned}
$$

While this equation has an appearance similar to Eq. (22), its meaning is different since the integrals are done according to the connectivity (in $\mathcal{V}$ ). This extra information permits us to group the different terms together. With $\mathrm{d} \Phi_{a_{+}}=\mathrm{d} \Phi_{a_{-}}=\mathrm{d} \Phi_{a}$ and $\mathrm{d} \Phi_{c_{+}}=\mathrm{d} \Phi_{c_{-}}=\mathrm{d} \Phi_{c}$, and the notation $\boldsymbol{r}_{i j}=\boldsymbol{x}_{j}-\boldsymbol{x}_{i}$ the above expression simplifies to:

$$
\begin{aligned}
\frac{\mathrm{d} H}{\mathrm{~d} t}= & \frac{1}{2 \pi} \int_{\Phi} \int_{\Phi}\left(\frac{\mathrm{d} \theta\left(\boldsymbol{r}_{c_{+} a_{-}}\right)}{\mathrm{d} t}+\frac{\mathrm{d} \theta\left(\boldsymbol{r}_{c_{-} a_{+}}\right)}{\mathrm{d} t}\right. \\
& \left.-\frac{\mathrm{d} \theta\left(\boldsymbol{r}_{c_{+} a_{+}}\right)}{\mathrm{d} t}-\frac{\mathrm{d} \theta\left(\boldsymbol{r}_{c_{-} a_{-}}\right)}{\mathrm{d} t}\right) \mathrm{d} \Phi_{a} \mathrm{~d} \Phi_{c} .
\end{aligned}
$$

We can now define a density of helicity flux for each flux tube; but this density is per unit of magnetic flux, not per unit surface. Thus for the flux tube labeled $a$ we have:

$$
\begin{aligned}
\left.\frac{\mathrm{d} h_{\Phi}}{\mathrm{d} t}\right|_{a}= & \frac{1}{2 \pi} \int_{\Phi}\left(\frac{\mathrm{d} \theta\left(\boldsymbol{r}_{c_{+} a_{-}}\right)}{\mathrm{d} t}+\frac{\mathrm{d} \theta\left(\boldsymbol{r}_{c_{-} a_{+}}\right)}{\mathrm{d} t}\right. \\
& \left.-\frac{\mathrm{d} \theta\left(\boldsymbol{r}_{c_{+} a_{+}}\right)}{\mathrm{d} t}-\frac{\mathrm{d} \theta\left(\boldsymbol{r}_{c_{-} a_{-}}\right)}{\mathrm{d} t}\right) \mathrm{d} \Phi_{c}
\end{aligned}
$$

It takes into account the relative motions of all the other flux tubes with respect to flux tube $a$. The total flux can be written as the integral of the flux density:

$\frac{\mathrm{d} H}{\mathrm{~d} t}=\left.\int_{\Phi} \frac{\mathrm{d} h_{\Phi}}{\mathrm{d} t}\right|_{a} \mathrm{~d} \Phi_{a}$

One can relate $\mathrm{d} h_{\Phi} / \mathrm{d} t$ to $G_{\theta}$ at both feet of flux tube $a$ if we separate in Eq. (25) the contributions at $\boldsymbol{x}_{a_{+}}$from those at $\boldsymbol{x}_{a_{-}}$ to get:

$\left.\frac{\mathrm{d} h_{\Phi}}{\mathrm{d} t}\right|_{a}=\frac{G_{\theta}\left(\boldsymbol{x}_{a_{+}}\right)}{\left|B_{n}\left(\boldsymbol{x}_{a_{+}}\right)\right|}+\frac{G_{\theta}\left(\boldsymbol{x}_{a_{-}}\right)}{\left|B_{n}\left(\boldsymbol{x}_{a_{-}}\right)\right|}$.

Then the helicity flux per unit magnetic flux, $\mathrm{d} h_{\Phi} / \mathrm{d} t$, is a field-weighted average of the flux per unit surface, $G_{\theta}$, at both footpoints.

While the helicity flux density has a meaning only for each individual flux tube, it is convenient to define a flux per unit surface area. This will enable us to compare helicity injection with other sets of observations (e.g. the location of emergence or of flaring). A helicity flux density per unit of surface, $B_{n} \mathrm{~d} h_{\Phi} / \mathrm{d} t$, can be assigned to either the positive or the negative polarity. Equivalently the helicity input in an elementary flux tube can be shared at both footpoints, e.g. setting a fraction $f_{+}=f$ in the positive polarity and $f_{-}=1-f$ in the negative polarity. Then we define the helicity flux density through $\mathcal{S}$ as:

$G_{\Phi}\left(\boldsymbol{x}_{a_{+}}\right)=\left.\frac{\mathrm{d} h_{\Phi}}{\mathrm{d} t}\right|_{a}\left|B_{n}\left(\boldsymbol{x}_{a_{+}}\right)\right| f_{+}$
$G_{\Phi}\left(\boldsymbol{x}_{a_{-}}\right)=\left.\frac{\mathrm{d} h_{\Phi}}{\mathrm{d} t}\right|_{a}\left|B_{n}\left(\boldsymbol{x}_{a_{-}}\right)\right| f_{-}$

where $0 \leq f \leq 1$ is a parameter that is chosen for convenience of representation. For example if we choose $f=1$ (resp. $f=$ 0 ), all the helicity flux per unit magnetic flux will be assumed to be injected through the positive (resp. negative) footpoint of each flux tubes. If $f=1 / 2$, one assumes that the helicity injection is equally shared by both footpoints.

One can relate $G_{\Phi}$ to $G_{\theta}$ at both feet of flux tube $a$ using Eqs. (27) and (28):

$G_{\Phi}\left(\boldsymbol{x}_{a_{ \pm}}\right)=\left(G_{\theta}\left(\boldsymbol{x}_{a_{ \pm}}\right)+G_{\theta}\left(\boldsymbol{x}_{a_{\mp}}\right)\left|\frac{B_{n}\left(\boldsymbol{x}_{a_{ \pm}}\right)}{B_{n}\left(\boldsymbol{x}_{a_{\mp}}\right)}\right|\right) f_{ \pm}$. 
Then the helicity flux density $G_{\Phi}$ is a field-weighted average of the flux density $G_{\theta}$ at both footpoints of the coronal flux tube.

\section{Illustrative examples}

The expression $G_{\Phi}$ (Eq. (28)) defines the correct helicity flux density. However accurately measuring $G_{\Phi}$ will certainly be difficult because coronal connectivities are only marked by a few loops. Moreover the ends of such loops are below coronal temperatures and so usually not observed. In addition, active region connectivity is usually complex, involving quasiseparatrix layers (QSLs): on both sides of a QSL field lines link very distant photospheric regions (Démoulin et al. 1997). Usually, only some flux tubes on one side of a QSL are dense enough to be observed. All these considerations imply that the use of $G_{\Phi}$ is practically limited by the available number of connectivities that observations can provide. Thus it is worth considering the densities $G_{A}$ (Eq. (13)) and $G_{\theta}$ (Eq. (19)) (which do not involve connectivity). Do these simpler densities provide measurements representative of the $G_{\Phi}$ distribution?

\subsection{Translational motion of a single magnetic polarity}

In observed magnetograms, magnetic flux tubes are frequently observed to move bodily, so we consider a simple circular magnetic region (with $B_{n}$ uniform, $=B_{0}$, in the disk of radius $R$ ) moving with a constant velocity $\boldsymbol{u}=U_{0} \hat{\boldsymbol{e}}_{x}$.

In this basic example, $G_{A}$ is positive in the top half of the region, and negative in the bottom half (Fig. 1):

$G_{A}(\boldsymbol{x})=y U_{0} B_{0}^{2} \quad$ for $|\boldsymbol{x}(x, y)| \leq R$.

Since $G_{A}$ is antisymmetric with respect to the $x$-axis, the helicity flux density is equal to 0 , as it should be since there is no helicity injection with this model. However, this clearly shows that one must be careful when directly interpreting any $G_{A}$ map: one could conclude wrongly that there is an injection/ejection of helicity while there is no helicity flux at all.

The total amount of helicity in each polarity of $G_{A}$ is large. For both polarities the total absolute flux is:

$\frac{\mathrm{d} H_{A,+}}{\mathrm{d} t}=\left|\frac{\mathrm{d} H_{A,-}}{\mathrm{d} t}\right|=\frac{2}{3} U_{0} B_{0}^{2} R^{3}$.

This can be compared to the helicity flux injected in one polarity (of flux $\Phi$ ) rotating rigidly with an angular velocity $\Omega=U_{R} / R\left(U_{R}\right.$ is the velocity at the polarity border):

$\frac{\mathrm{d} H_{\mathrm{rot}}}{\mathrm{d} t}=\frac{\Omega}{2 \pi} \Phi^{2}=\frac{\pi}{2} U_{R} B_{0}^{2} R^{3}$,

then

$\frac{\mathrm{d} H_{A,+} / \mathrm{d} t+\left|\mathrm{d} H_{A,-} / \mathrm{d} t\right|}{\mathrm{d} H_{\text {rot }} / \mathrm{d} t}=\frac{8}{3 \pi} \frac{U_{0}}{U_{R}}$.

So the total absolute flux falsely computed with $G_{A}$ is similar to the flux injected by twisting motions when the translation motion is comparable to the rotation velocity $\left(U_{0} \approx U_{R}\right)$ !

The above spurious contribution of $G_{A}$ can easily mask the true helicity flux density, especially because the values of $U_{R}$ are expected to be substantially lower than those of $U_{0}$.
For example, with a twist of one turn in 10 days, $U_{R}$ will be in the interval $[0.005,0.05] \mathrm{km} \mathrm{s}^{-1}$ with $R$ belonging to $\left[10^{3}, 10^{4}\right] \mathrm{km}$; meanwhile $U_{0}$ is frequently observed to be of the order of several $0.1 \mathrm{~km} \mathrm{~s}^{-1}$ (e.g. maximum values 0.3 to $0.7 \mathrm{~km} \mathrm{~s}^{-1}$ for the ARs analyzed by Nindos et al. 2003). The maximum rotation velocities deduced from the best examples of rotating asymmetric sunspots are in the interval $[0.03,0.1] \mathrm{km} \mathrm{s}^{-1}$ (Brown et al. 2003), a range which is just around the maximal magnitude estimated above for $U_{R}$. Thus $U_{0}$ is expected to dominate $U_{R}$ in most ARs. Moreover, tracking methods detect translational motions much more easily than rotating ones (Démoulin \& Berger 2003; Gibson et al. 2004), so it is vital to not include the strong translational signal in the helicity flux density.

In the above case of the translation motion of a single magnetic polarity, both $G_{\theta}$ and $G_{\Phi}$ solve the problem perfectly: since the rotation rate $\mathrm{d} \theta\left(\boldsymbol{x}-\boldsymbol{x}^{\prime}\right) / \mathrm{d} t$ is null for every pairs of elementary fluxes, we have

$G_{\theta}(\boldsymbol{x})=G_{\Phi}(\boldsymbol{x})=0$.

\subsection{Two separating magnetic polarities}

\subsection{1. $G_{A}$ and $G_{\theta}$ distributions}

At the photospheric level, magnetic flux tubes move relative to each other. A basic motion is the separation of two opposite polarities (e.g. after emergence of a bipole). For concreteness, we consider two circular opposite polarities $\left(P_{+}\right.$and $\left.P_{-}\right)$which separate without any rotation (so there is no helicity injection). Their velocities are:

$\boldsymbol{u}_{-}=-\boldsymbol{u}_{+}=U_{0} \hat{\boldsymbol{e}}_{x}$

Let $O_{+}\left(O_{-}\right)$be the position of the centre of the positive (resp. negative) polarity with $\boldsymbol{O}_{+} \boldsymbol{O}_{-}=D \hat{\boldsymbol{e}}_{x}$. The following results can also be applied to magnetic polarities approaching each others (there is only a sign change for $G_{A}$ and $G_{\theta}$ ).

The values of $G_{A}$ and $G_{\theta}$ at the point $M(x)$ are:

$G_{A}(\boldsymbol{x})= \pm y U_{0} B_{0}^{2}\left(1-\frac{R^{2}}{\left|O_{ \pm} M\right|^{2}}\right) \quad$ for $M \in P_{\mp}$

$G_{\theta}(\boldsymbol{x})=\mp y U_{0} B_{0}^{2} \frac{R^{2}}{\left|O_{ \pm} M\right|^{2}} \quad$ for $M \in P_{\mp}$.

Both $G_{A}$ and $G_{\theta}$ present non zero values (Fig. 2). As explained at the end of Sect. 3.2, $G_{\theta}$ presents fake polarities due to a nonzero relative rotation rate. This clearly shows that neither $G_{A}$ nor $G_{\theta}$ are the real helicity flux density. But $G_{\theta}$ gives much lower values than $G_{A}$ : the ratio between $G_{A}$ and $G_{\theta}$ is equal to:

$\frac{G_{A}}{G_{\theta}}=1-\frac{\left|O_{ \pm} M\right|^{2}}{R^{2}} \quad$ for $M \in P_{\mp}$,

and so roughly only depends of the ratio $D / R$ (we have $G_{A} / G_{\theta} \approx 1-(D / R)^{2}$ for $\left.D \gg R\right)$. For example, with $D=10$ and $R=3$ (Fig. 2) we get $G_{A}>10 G_{\theta}$. At the limit $D / R \rightarrow \infty$, Eqs. (36) and (37) respectively reduce to Eqs. (30) and (34) for an isolated magnetic polarity. 


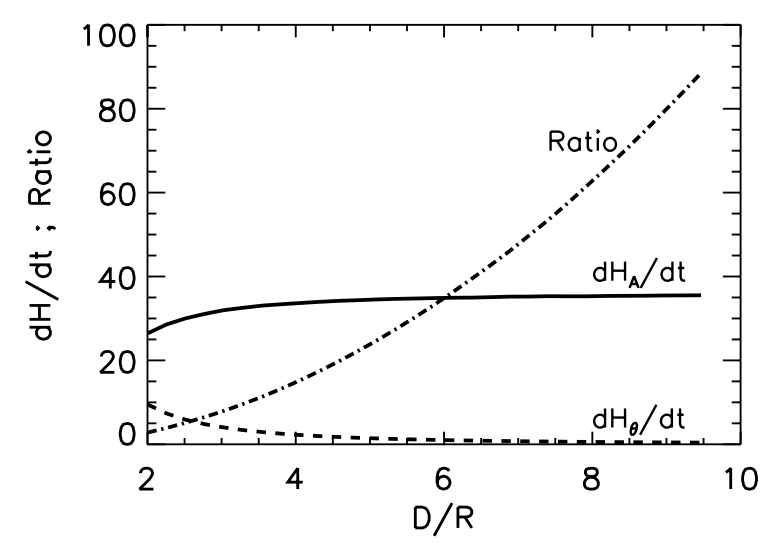

Fig. 4. Total positive fluxes for $G_{A}$ and $G_{\theta}$ for two separating magnetic regions as in Fig. 2. The total fluxes $\left(\mathrm{d} H_{A} / \mathrm{d} t\right)_{+}$(with $G_{A}>0$, Eq. (39)), $\left(\mathrm{d} H_{\theta} / \mathrm{d} t\right)_{+}$(with $G_{\theta}>0$, Eq. (40)) and their ratio are given in function of the separation distance $D$ normalized to the radius $R$ of the magnetic regions (for $D / R=2$ the magnetic regions are in tangential contact). As $D / R$ increases, $\left(\mathrm{d} H_{\theta} / \mathrm{d} t\right)_{+}$decreases rapidly towards zero, while $\left(\mathrm{d} H_{A} / \mathrm{d} t\right)_{+}$saturates (giving the case of Fig. 1 for each magnetic region). The fluxes are drawn with $U_{0} B_{0}^{2} R^{3}=27$.

The total positive helicity fluxes of each magnetic polarity are:

$$
\begin{aligned}
\left(\frac{\mathrm{d} H_{A}}{\mathrm{~d} t}\right)_{+, 1 \text { pol. }} & =\int_{\mathcal{S}, G_{A}>0} G_{A} \mathrm{~d} \mathcal{S} \\
& =U_{0} B_{0}^{2} R^{3}(-1 / 3+f(R / D)) \\
& =\int_{\mathcal{S}, G_{\theta}>0} G_{\theta} \mathrm{d} \mathcal{d} \\
& =U_{0} B_{0}^{2} R^{3}(1-f(R / D)) \\
\text { with } f(x) & =\frac{1-x^{2}}{2 x} \ln \left(\frac{1+x}{1-x}\right) .
\end{aligned}
$$

For $D \gg R$ we have approximately:

$$
\left(\frac{\mathrm{d} H_{A}}{\mathrm{~d} t}\right)_{+, 1 \text { pol. }} \approx \frac{2}{3} U_{0} B_{0}^{2} R^{3}\left(1-(R / D)^{2}\right)
$$

$$
\left(\frac{\mathrm{d} H_{\theta}}{\mathrm{d} t}\right)_{+, 1 \text { pol. }} \approx \frac{2}{3} U_{0} B_{0}^{2} R^{3}(R / D)^{2}
$$

so that, as the relative distance $D / R$ between polarities increases, $\left(\mathrm{d} H_{A} / \mathrm{d} t\right)_{+}$tends to a constant (Eq. (31)) while $\left(\mathrm{d} H_{\theta} / \mathrm{d} t\right)_{+}$decreases rapidly toward zero $\left(\right.$as $\left.(R / D)^{2}\right)$. In the worst case for $G_{\theta}$, when the polarities are into contact $(D=$ $2 R$ ), the spurious unsigned total helicity flux given by $G_{\theta}$ is a factor $\sim 3$ lower than the flux derived by using $G_{A}$ (Fig. 4). But when the borders of the polarities are only separated by their diameter (so $D=4 R$ ) this factor reaches $\sim 15$ !

\subsection{2. $G_{\phi}$ distributions}

The helicity flux density defined by the linkage, $G_{\Phi}$, averages the values of $G_{\theta}$ at both footpoints of each flux tube (Eq. (29)). For a potential field connection between the two moving magnetic regions we have: $G_{\theta}\left(\boldsymbol{x}_{a_{+}}\right)=-G_{\theta}\left(\boldsymbol{x}_{a_{-}}\right)$ and $B_{n}\left(\boldsymbol{x}_{a_{+}}\right)=\left|B_{n}\left(\boldsymbol{x}_{a_{-}}\right)\right|$, which implies $\mathrm{d} h_{\Phi} /\left.\mathrm{d} t\right|_{a}=0$ (Eq. (27)) and $G_{\Phi}\left(\boldsymbol{x}_{a_{+}}\right)=G_{\Phi}\left(\boldsymbol{x}_{a_{-}}\right)=0$ (Eq. (29)). There is no helicity flux density as expected.

However when the magnetic polarities are linked by a twisted flux tube, there is in general a non null flux density as follows. For simplicity, suppose that the flux tube, which links the magnetic regions, has a uniform twist $T$ in its cross-section. Let $T \geq 0$ be fixed as the magnetic polarities separate (so there is no twisting motions and no net injection of magnetic helicity) and analyze the effect of $T$ on the distribution of $G_{\Phi}$. For $T>0$, $G_{\theta}\left(\boldsymbol{x}_{a_{+}}\right) \neq-G_{\theta}\left(\boldsymbol{x}_{a_{-}}\right)$and the difference increases from $T=0$ to $T=1 / 2$ (for a half turn $G_{\theta}\left(\boldsymbol{x}_{a_{+}}\right)=G_{\theta}\left(\boldsymbol{x}_{a_{-}}\right)$). Thus $G_{\Phi}$ depends on the amount of twist in the volume. More precisely, taking $f_{+}=f_{-}=0.5$ (so sharing the flux equally in each magnetic polarity) $G_{\Phi}$ is in the interval $\left[0, G_{\theta}\right]$ when $T$ is in $[0,1 / 2]$ !

The twist $T$ indeed creates an asymmetry between the elementary fluxes linking the polarities. Let us call $\mathcal{A}$ the field line linking the centre of the polarities (axis of the torus). When $T$ is in $[0,1 / 2]$, part of the flux linkage lies below $\mathcal{A}$ while the other part lies above $\mathcal{A}$ and at $T=1 / 2$, this effect is maximum. As the magnetic polarities separate, the elementary flux tubes going above (resp. below) $\mathcal{A}$ get a magnetic helicity flux which increases (resp. decreases) their positive helicity. Although there is no net global flux of helicity, the footpoint separation creates a reorganization in the helicity distribution and thus the appearance of positive/negative helicity flux densities. For $T>1 / 2, G_{\Phi}$ decreases and goes to zero for $T=1$ since all the flux is now passing below $\mathcal{A}$. Finally, the distribution of $G_{\Phi}$ is modified periodically with $T$ with a period of 1 . This example illustrates that the remnant polarities in $G_{\theta}$ can be artificial or real depending on the coronal linkage.

\subsubsection{Effects of $B_{n}$ distributions}

We have assumed above a uniform field component $B_{n}$ in the magnetic regions. We investigate below how profile dependent the above result is. The selected profiles are ordered from a profile where the flux is mostly at the border of the magnetic region to a profile where the flux is concentrated around the centre of the region (keeping the same total magnetic flux):

$$
\begin{aligned}
& B_{1}(\rho)=\frac{3 \rho}{2 R} B_{0} \\
& B_{2}(\rho)=B_{0} \\
& B_{3}(\rho)=\frac{B_{0}}{\ln 2\left(1+(\rho / R)^{2}\right)} \\
& B_{4}(\rho)=3 B_{0}(1-\rho / R) \\
& B_{5}(\rho)=\frac{1-\rho / R}{(3-4 \ln 2)(1+\rho / R)}
\end{aligned}
$$

where $\rho$ is the distance from the centre $(\rho \leq R)$. The ratio between $\left(\mathrm{d} H_{A} / \mathrm{d} t\right)_{+}$and $\left(\mathrm{d} H_{\theta} / \mathrm{d} t\right)_{+}$shows that $G_{\theta}$ introduces, relatively to $G_{A}$, even less spurious helicity flux when the flux is more concentrated (Fig. 5). Only when the magnetic flux lies mostly at the border of the magnetic region (field $B_{1}$ in Eq. (41)) will the ratio be slightly less than in the case where $B_{n}$ is uniform. Since profiles of observed photospheric flux tubes show $B_{n}$ decreasing from the centre to the border, the case with 

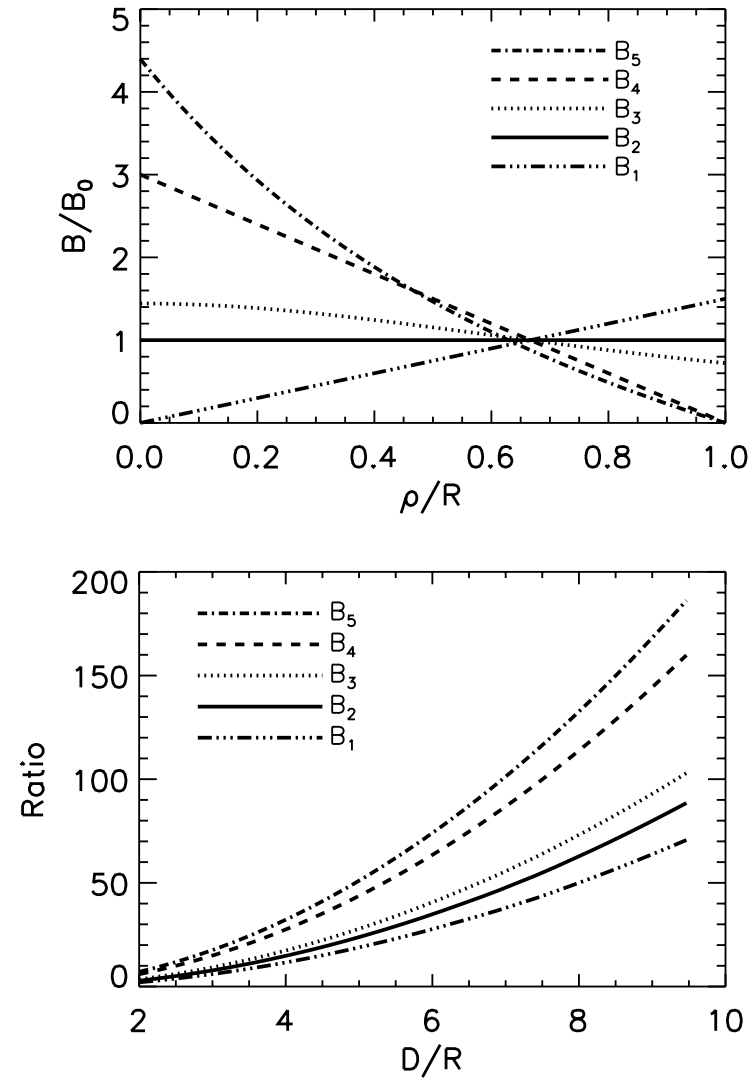

Fig. 5. Top panel: different profiles of $B_{n}(\rho / R)$ as described by Eq. (41). Bottom panel: ratio of $\left(\mathrm{d} H_{A} / \mathrm{d} t\right)_{+}$(Eq. (39)) with $\left(\mathrm{d} H_{\theta} / \mathrm{d} t\right)_{+}$ (Eq. (40)) for the different $B_{n}$ profile in function of the separation distance $D$ normalized to the radius $R$ of the magnetic regions. The ratio is larger as the field is more concentrated to the centre of the magnetic region.

a uniform $B_{n}$ provides a good estimate of the strongest spurious helicity flux given with $G_{\theta}$.

\subsubsection{Case of magnetic regions with like-sign}

Finally, consider the same configuration as above except that the two magnetic regions have the same $B_{n}$ sign. The main difference is that the - sign in Eq. (36) is replaced by a + sign (also $G_{\theta}$ changes sign in Eq. (37)). It implies that Eq. (38) is replaced with a summation of two terms of like sign, so the ratio $\left|G_{A} / G_{\theta}\right|$ is always greater. Thus in the case of two magnetic regions of same sign the use of $G_{\theta}$, rather than $G_{A}$, is even more recommended.

\subsection{One polarity rotating around another one}

Another basic motion is the rotation of one magnetic region around another one. For concreteness we consider that the magnetic region $P_{-}$is rotating around the magnetic region $P_{+}$, but for the following helicity flux results the field sign can be reversed. Each magnetic region is circular with a radius $R$ and $\left|B_{n}\right|=B_{0}$ uniform. The centres of the polarities, $O_{+}$and $O_{-}$, are separated by $D=\left|O_{+} O_{-}\right|$. We suppose that only $P_{-}$has a solid rotation around $P_{+}$with the angular velocity $\boldsymbol{\Omega}=\Omega \hat{\boldsymbol{e}}_{x}$; the velocity field at the point $M(\boldsymbol{x})$ is given by:

$\boldsymbol{u}=\boldsymbol{\Omega} \times \boldsymbol{O}_{+} M \quad$ for $M \in P_{-}$.

In this example there is a net total flux of helicity. The rigid rotation of $P_{-}$around $P_{+}$can be decomposed into a rigid rotation of $P_{-}$around its centre and a rotation of $P_{-}$around $P_{+}$ without internal rotation of $P_{-}$(see Eq. (A.1)). Thus the helicity injected has three contributions: $\dot{T}_{-}$due to the rotation of $P_{-}$ around itself (twisting), $\dot{W}_{-+}$due to the relative rotation of $P_{-}$ around $P_{+}$(writhe) and $\dot{W}_{+-}$due to the relative rotation of $P_{+}$around $P_{-}$(writhe). Since all these rotations have the same rotation rate $\Omega$, using Eq. (23) and assuming that the two polarities are elementary fluxes (point-like with $\Phi_{-}=\Phi_{+}=\pi R^{2} B_{0}$ a fixed value and $D \gg R$ ), the total flux of helicity injected is:

$$
\begin{aligned}
\frac{\mathrm{d} H}{\mathrm{~d} t} & =\dot{W}_{-+}+\dot{W}_{+-}+\dot{T}_{-} \\
& =\frac{\Omega}{2 \pi}\left(\Phi_{-} \Phi_{+}+\Phi_{+} \Phi_{-}-\Phi_{-}^{2}\right) \\
& =\frac{\pi}{2} \Omega B_{0}^{2} R^{4} .
\end{aligned}
$$

The non-null values of $G_{A}$ and $G_{\theta}$ are:

$$
\begin{aligned}
& G_{A}(\boldsymbol{x})=\Omega B_{0}^{2}\left(R^{2}-\boldsymbol{O}_{-} \boldsymbol{M} \cdot \boldsymbol{O}_{+} \boldsymbol{M}\right) \quad \text { for } M \in P_{-} \\
& G_{\theta}(\boldsymbol{x})=\frac{\Omega B_{0}^{2} R^{2}}{2} \frac{\boldsymbol{O}_{-} \boldsymbol{M} \cdot \boldsymbol{O}_{-} \boldsymbol{O}_{+}}{\left|O_{-} M\right|^{2}} \text { for } M \in P_{+} .
\end{aligned}
$$

$G_{A}$ vanishes in $P_{+}$because $P_{+}$is not moving. However it is worth noting that $G_{\theta}$ vanishes in $P_{-}$! At each point of $P_{-}$the twist helicity is exactly compensated by the writhe helicity. The detail of the $G_{\theta}$ computation which leads to Eq. (45) is given in Appendix A. Integration of both Eqs. (44) and (45) over $P_{-}$ and $P_{+}$respectively gives the result of Eq. (43), showing that this result applies also for any finite radius $R$.

Like when two polarities separate (Sect. 4.2), $G_{A}$ has both positive and negative values on the rotating region, a result which could lead to misinterpretation (Fig. 6). At the opposite of the separating case, $G_{\theta}$ is positive in all of the motionless region $\left(P_{+}\right)$. The possible misinterpretation in that case is that the helicity flux is concentrated in $P_{+}$. Using $G_{\Phi}$ (Eq. (28)) solves this problem.

When $D \gg R, G_{\theta} \approx \Omega B_{0}^{2} R^{2} / 2$ is almost uniform in $P_{+}$ which is just what is expected for a solid rotation. However $G_{A}$ is growing with the separation, $D$, of the magnetic polarities. For $D \gg R$, the ratio between $G_{A}$ in $P_{-}$and $G_{\theta}$ in $P_{+}$is approximately:

$\frac{G_{A}}{G_{\theta}} \approx 2 \frac{D}{R} \cos \varphi$

where $\cos \varphi=\boldsymbol{O}_{-} \boldsymbol{M} \cdot \boldsymbol{O}_{-} \boldsymbol{O}_{+} /(R D)$ for $M \in P_{-}$. Then the fake polarities of $G_{A}$ grows linearly with the separation $D$ of the magnetic regions! The total helicity fluxes, computed separately for positive and negative densities as in Eqs. (39) and (40), have the same properties: $\left(\mathrm{d} H_{A} / \mathrm{d} t\right)_{+}$and $\left|\left(\mathrm{d} H_{A} / \mathrm{d} t\right)_{-}\right|$ both grow linearly with $D / R$ while $\left(\mathrm{d} H_{\theta} / \mathrm{d} t\right)_{+}$gives the right input of helicity (Fig. 7).

Furthermore, the sum $\left(\mathrm{d} H_{A} / \mathrm{d} t\right)_{+}+\left(\mathrm{d} H_{A} / \mathrm{d} t\right)_{-}$gives only approximately the correct injected helicity; the error grows 

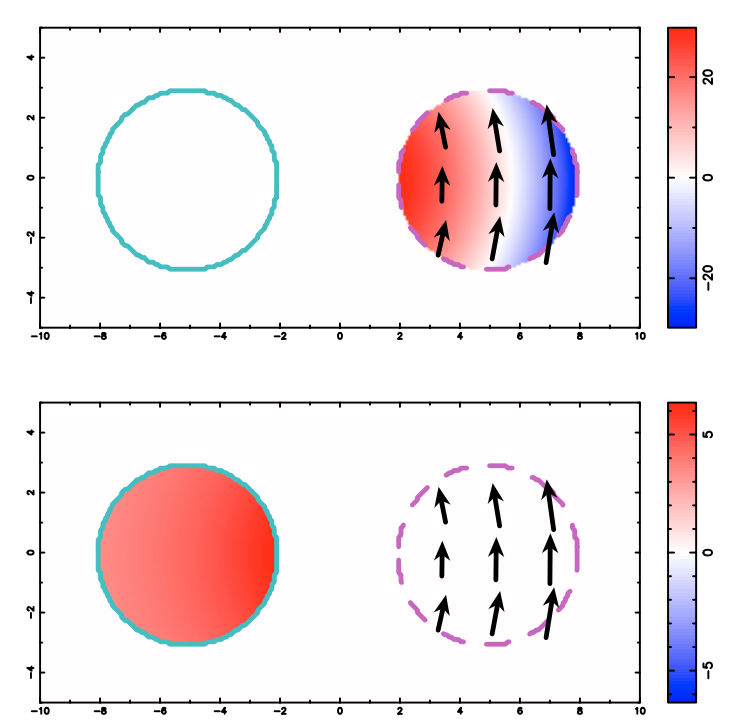

Fig. 6. Maps of $G_{A}$ (Eq. (13), top panel) and $G_{\theta}$ (Eq. (19), bottom panel) in the case of one magnetic polarity $\left(P_{-}\right)$having a solid rotation around another one $\left(P_{+}\right)$and injecting positive magnetic helicity (motions are indicated with arrows). $B_{n}$ is uniform in each magnetic region. $G_{A}$ is strongly bipolar while $G_{\theta}$ has no negative value. The shading convention is the same as in Fig. 1 (a color version is available in the electronic version at http://www.edpsciences.org).

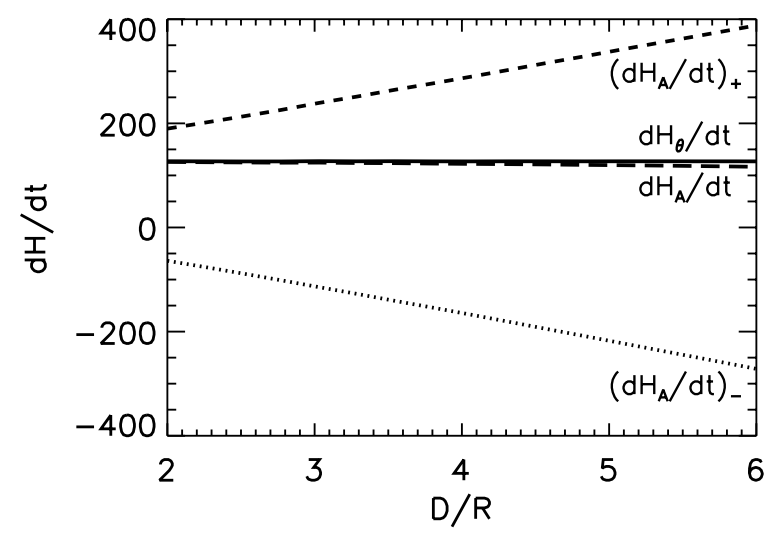

Fig. 7. Total positive and negative helicity flux for $G_{A}$ and total positive flux for $G_{\theta}$ (no negative values) in the case of one magnetic region having a solid rotation around another one (Fig. 6). The abscissa is the relative distance $D / R$ as in Fig. 5. The fake flux, both positive and negative, given by $G_{A}$ is of comparable magnitude to the real flux $\left(\Omega B_{0}^{2} R^{4}=81\right)$.

with $D / R$. It shows that, not only the local density values, but also the total helicity flux can be affected by using $G_{A}$. Large positive and negative fluxes are cancelled in the total flux; moreover the computation of $\boldsymbol{A}$ is affected by the periodicity of the fast Fourier transform (used to compute it). In the present case the periodic boundary effect is dominant and we describe it below.

In order to accurately compute $\boldsymbol{A}$ using the fast Fourier transform method, one must place the magnetogram in a larger field of view that contains no magnetic field. In this paper, the data box was a $512 \times 512$ mesh representing a region of $40 \times$ 40. To compute $\boldsymbol{A}$ we put this data box in the centre of a $4 \times$ 4 times larger box. Thus the fast fourier transform is here done with $2048 \times 2048$ modes, a much larger number of modes than what is usually used with observations. This large number reduces the effect of the periodic boundary here (so that it is small in Fig. 7). This effect would become even more important with real observations and when the polarities are well separated. This is another important point in favor of $G_{\theta}$ since only $G_{A}$ is affected by this numerical problem.

\subsection{Emergence of a twisted flux tube}

\subsubsection{Description of the model}

Frequently new bipoles appear in magnetograms. The associated magnetic flux tube is expected to be twisted in the convective zone in order to be able to reach the photosphere without being destroyed (Emonet \& Moreno-Insertis 1998). There is also evidence for the emergence of twisted flux tubes (Leka et al. 1996).

We consider a simple model to simulate the main helicity properties of this emergence. The magnetic field is confined in a torus with a main axial radius $R_{\mathrm{a}}$ and a small radius $R$. The longitudinal field strength inside the torus is uniform for simplicity (similar results are obtained with a different profile, as in Fig. 5). The field is uniformly twisted, both across and along the flux tube, with a number $N>0$ of turns for half the torus.

We simulate the emergence of half the torus with a constant vertical velocity. The horizontal velocity of the footpoints of field lines is given by Eq. (7), with no horizontal plasma velocity. The helicity flux is thus given by Eq. (12). The height of the axis apex above the "photosphere" (boundary) is used to monitor the emergence. Its value is $-R$ when the first amount of field is about to cross the "photosphere", 0 , when the torus axis is just tangent to the "photosphere", $R$ when the top of the torus (its central cross section) has completely emerged and $R_{\mathrm{a}}$ when the torus is half emerged. Normalizing the height with $R_{\mathrm{a}}$, the relative height $Z$ runs from $-R / R_{\mathrm{a}}$ to 1 .

\subsubsection{General properties}

As in the other examples, $G_{A}$ and $G_{\theta}$ have some negative helicity flux densities while the rising of this twisted flux tube should only inject positive helicity (Fig. 8). Since the magnetic field is known in the volume, we know the connectivities and we can compute $G_{\Phi}$ (Fig. 8). $G_{\Phi}$ has no negative polarities and it is almost homogeneous over the flux tube section. The evolution of the helicity flux during the emergence is summarized by the total fluxes (Fig. 9). All total helicity injections are maximum just before $Z=R / R_{\mathrm{a}}$, i.e. just before the top of the flux tube has completely emerged. The helicity injections are related to the modification of the flux-tube cross section with $Z$, called the Cassini surface, but cannot be expressed only as a function of this surface (even with a uniform longitudinal field and twist as considered here).

For cases with low $N$ even $G_{\theta}$ maps have fake polarities but they are at least a factor 10 lower than the fake polarities of $G_{A}$. For example, with a flux tube twisted by only one tenth of turn $(N=0.1)$ the fake polarities of $G_{\theta}$ are of the same magnitude 

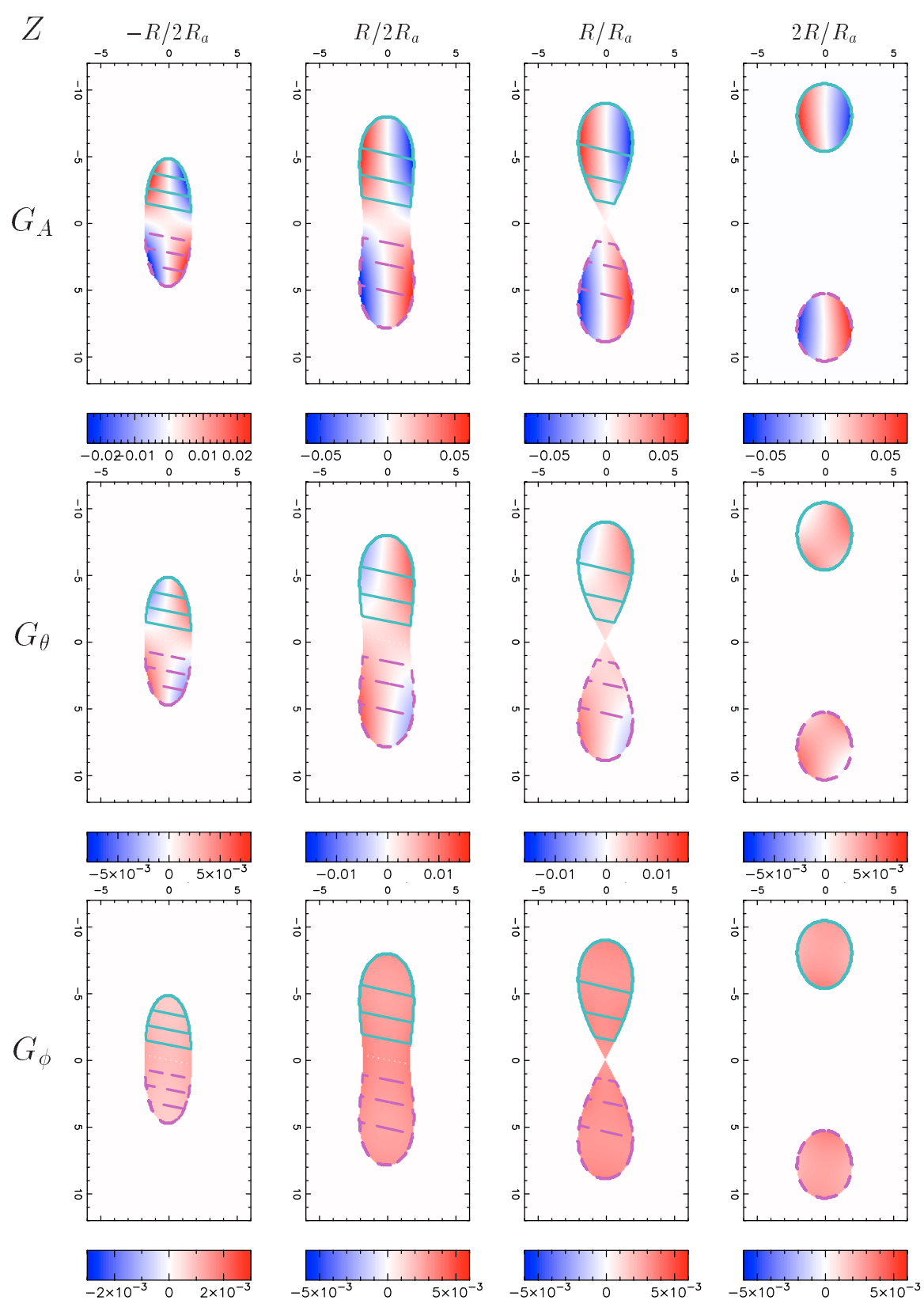

Fig. 8. Rows from top to bottom: maps of $G_{A}, G_{\theta}, G_{\Phi}$ (Eqs. (13), (19) and (28) with $f=1 / 2$ ) in the case of the emergence of a twisted flux tube (as defined in Sect. 4.4). The helicity flux densities are presented at four different times with the flux tube rising from left to right. The number of turns in half the torus is $N=0.1$, and the aspect ratio $R / R_{\mathrm{a}}=0.2$. The shading convention is the same as in Fig. 1 with a range which depends on the panel (a color version is available in the electronic version at http://www. edpsciences.org). The continuous (dashed) lines are positive (negative) isocontours of $B_{n}$, respectively.

than the real flux values given by the $G_{\Phi}$ maps (see the scales in the bottom of the maps of Fig. 8), while the fake polarities of $G_{A}$ are completely masking the real helicity injection during almost all the emergence (except when the torus is nearly half emerged: Fig. 9).

\subsubsection{Parametric study of the fake polarities}

As in the case of a single polarity translating and rotating (see Sect. 4.1 and Eq. (33)), the relative importance of the fake polarities depends of the amount on helicity injected (so on the number of turns $N$ ) and on the aspect ratio $R / R_{\mathrm{a}}$. We define a proxy, called the Fake Relative Flux $\left(\mathrm{FRF}_{X}\right)$, to quantify the relative importance of the fake polarities due to the use of $G_{X}$ (where $X=A$ or $\theta$ ):

$\mathrm{FRF}_{X}=\frac{\int_{Z=-R / R_{\mathrm{a}}}^{R / R_{\mathrm{a}}}\left|\frac{\mathrm{d} H_{X}}{\mathrm{~d} t}\right|_{-} \mathrm{d} Z}{\int_{Z=-R / R_{\mathrm{a}}}^{R / R_{\mathrm{a}}}\left|\frac{\mathrm{d} H_{\Phi}}{\mathrm{d} t}\right| \mathrm{d} Z}$.

The integral range is selected to be for the emergence of the top part of the flux tube, where the strongest fake polarities 


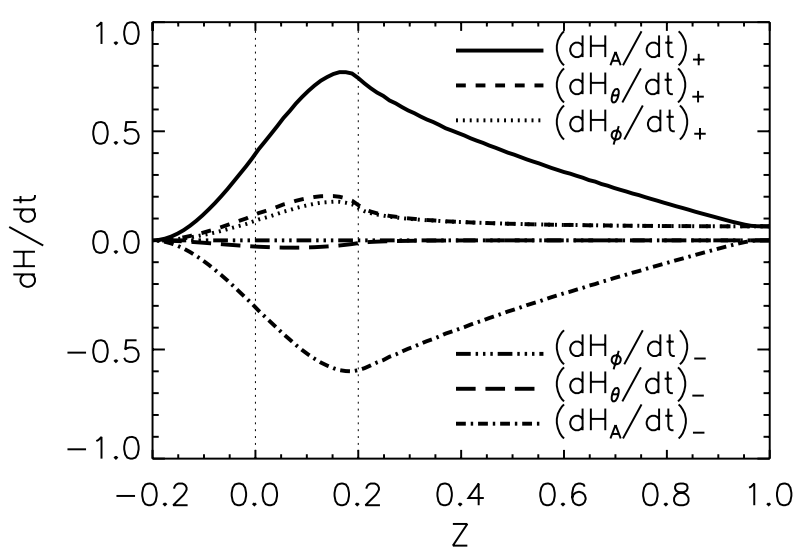

Fig. 9. Total positive and negative helicity flux for $G_{A}, G_{\theta}$, and $G_{\Phi}$ in the case of the emergence of a twisted flux tube with $N=0.1$ (Fig. 8). The abscissa is the relative height, $Z$, of the central part of the torus, above the "photosphere". The curves start (on the left) when the top of the torus crosses the "photosphere" $\left(Z=-R / R_{\mathrm{a}}=-0.2\right)$ and end (right) when the torus is half emerged $(Z=1)$. The thin vertical dotted lines correspond to the height when the top of the tube (its central cross section) is half emerged $(Z=0)$ and completely emerged $\left(Z=R / R_{\mathrm{a}}=0.2\right)$.

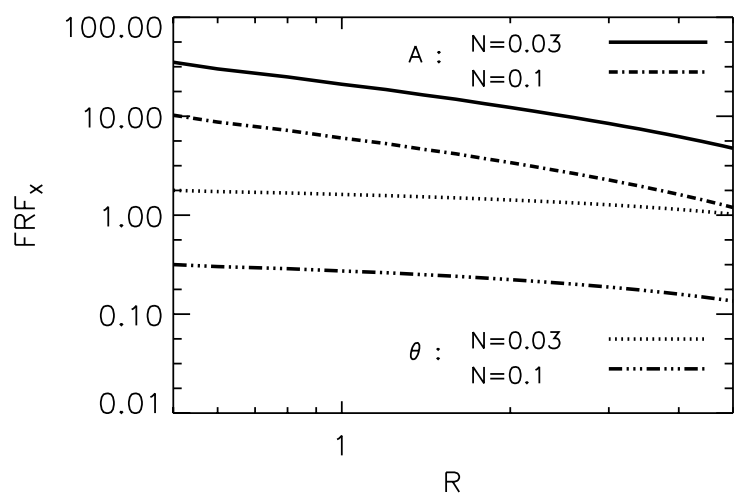

Fig. 10. Dependence on $R$ of the Fake Relative Fluxes, $\mathrm{FRF}_{A}$ and $\mathrm{FRF}_{\theta}$ (ratio between the fake and the real helicity flux for $Z \in$ $\left[-R / R_{\mathrm{a}}, R / R_{\mathrm{a}}\right]$, Eq. (47)). The curves show two values of the number of turns $N$ : 0.03 and 0.1 .

are present, but the following conclusion is the same with another choice. When $\mathrm{FRF}_{X}>1$, the fake polarities become more important than the true helicity flux and the maps of $G_{X}$ are misleading, while when $\mathrm{FRF}_{X} \ll 1$, the fake polarities do not significantly alter the true helicity flux density. We computed $\mathrm{FRF}_{A}$ and $\mathrm{FRF}_{\theta}$ for different values of $N$ ranging from 0.005 to 5 , and $R$ going from 0.5 up to 5 , keeping $R_{\mathrm{a}}$ constant equal to 10 . The results are summarized below.

Both $\mathrm{FRF}_{A}$ and $\mathrm{FRF}_{\theta}$ are decreasing functions of $R$ (Fig. 10), but the variation is relatively small, in particular with $\mathrm{FRF}_{\theta}$ which is only lightly influenced by $R$ (less than a factor 3 of variation for one order of magnitude in $R$ ). $\mathrm{FRF}_{A}$ is slightly more dependent on $R$ and its variation goes approximately as $R^{-1}$. However, $R$ (or the aspect ratio $R / R_{\mathrm{a}}$ ) is not a parameter as important as $N$ (see next paragraphs), and thus, for helicity injection maps coming from observations, the flux measurements in the large flux tubes will only be slightly less affected by the fake polarities than smaller flux tubes.

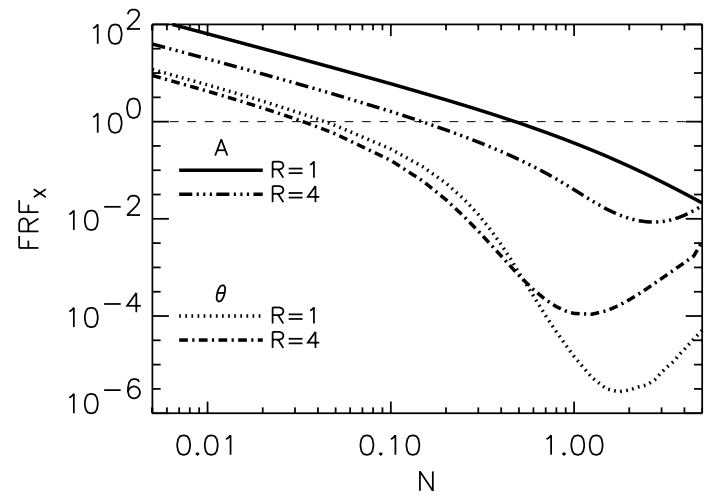

Fig. 11. Dependence on the number of turns $N$ of the Fake Relative Fluxes $\left(\mathrm{FRF}_{A}\right.$ and $\mathrm{FRF}_{\theta}$, Eq. (47)) for two values of the small radius $R$ : 1 and 4 (the main radius is $R_{\mathrm{a}}=10$ ).

The main effects of $N$ are the following ones. For small $N$, both $\mathrm{FRF}_{A}$ and $\mathrm{FRF}_{\theta}$ are rapidly decreasing functions of $N$ (more rapidly than $N^{-2}$ ), but for large $N$ they are increasing functions of $N$ (Fig. 11). The minimum point is located at a lower $N$ for $\mathrm{FRF}_{\theta}$ than for $\mathrm{FRF}_{A}$ (as well as for larger $R$ ).

The decrease for small $N$ can be easily understood. The total amount of helicity injected increases with $N$ while the separation velocity of the magnetic polarities, at the origin of the fake polarities, does not change. It implies that the fake polarities are more dominated by the real injected helicity at larger $N$ values. For example with $R / R_{\mathrm{a}}=4 / 10$ (Fig. 11), as soon as $N$ becomes bigger than $0.15, \mathrm{FRF}_{A}$ is lower than 1 . With lower radius, this transition happens for larger $N$. In the case of $\mathrm{FRF}_{\theta}$, whatever the radius is (in the range: $R / R_{\mathrm{a}} \in$ $[0.05,0.5]$ ), when $N>0.04$ (a low amount of twist), the fake polarities of $G_{\theta}$ do not mask the true helicity flux density.

The above dependence on $N$ is modified when $N$ is high enough so that it drastically changes the distribution of $B_{n}$ (Fig. 12). This occurs only at the top of the flux tube when $B_{n}$ is dominated by the azimuthal field. There, the two magnetic polarities are separating in a direction almost orthogonal to the torus axis (while they are separating nearly along the torus axis for smaller $N$ values, Fig. 12). The separation of the polarities leads to fake polarities of both $G_{A}$ and $G_{\theta}$ as in previous examples. Since in the case of separating polarities the strength of $G_{A}$ and $G_{\theta}$ depends on the magnetic vertical field and since in the top of the flux tube $B_{n} \propto N$, the importance of these fake polarities will increase with $N$. However, even in the case of these large values of $N$, the values of both $\mathrm{FRF}_{A}$ and $\mathrm{FRF}_{\theta}$ are still far below 1, implying a small influence of the fake polarities.

We conclude this emergence study with the hope of applying it to observations in a further study. The most important result is that, for the same evolving magnetic configuration, $\mathrm{FRF}_{\theta}$ is always lower by a factor 10 than $\mathrm{FRF}_{A}$, and this can be by more than a factor $10^{3}$ (for example with $R / R_{\mathrm{a}}=0.1$ and $N=1$ ). This strongly indicates that it is always better to use $G_{\theta}$ than $G_{A}$ in order to map the helicity flux density. Another important point is that with $G_{\theta}$ the fake polarities become negligible for lower values of $N$ than with $G_{A}$ (typically a factor 10 lower). 


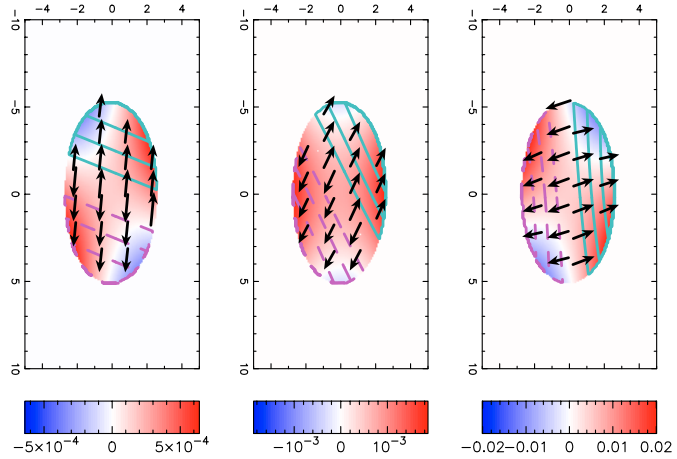

Fig. 12. Maps of $G_{\theta}$ (Eq. (19)) in the case of an emerging twisted flux tube for different number of turns $N$ of the field lines. From left to right, $G_{\theta}$ is drawn respectively with $N=0.2, N=1$ and $N=6$. The maps are for $Z=-3 R / 4 R_{\mathrm{a}}$ with $R / R_{\mathrm{a}}=0.4$. The arrows represent the horizontal motions $\boldsymbol{u}$ of the footpoints of the field lines (Eq. (7)). The shading convention is the same as in Fig. 1 with a range which depend on the panel (a color version is available in the electronic version at http://www.edpsciences.org). The continuous (dashed) line represents one isocontour of $B_{n}>0\left(B_{n}<0\right)$, respectively.

\section{Conclusion}

Magnetic helicity is a basic MHD quantity. Important developments have been achieved in recent years in observing the photospheric flux of magnetic helicity. It is also worthwhile to derive maps of the magnetic flux density in order to follow where the helicity is coming from (e.g. is it coming into the corona mainly in emerging magnetic fields or is a significant flux also present well after emergence?).

All previous analyses used the density $G_{A}\left(=-2(\boldsymbol{A} \cdot \boldsymbol{u}) B_{n}\right)$ which involves the vector potential $\boldsymbol{A}$ of the magnetic field (Chae 2001; Chae et al. 2001, 2004; Kusano et al. 2002, 2005; Nindos \& Zhang 2002; Nindos et al. 2003; Moon et al. $2002 \mathrm{a}, \mathrm{b})$. In all the studied active regions, the density $G_{A}$ has strong polarities of both sign with comparable magnitude. In fact, we show above that a large part of these polarities are likely to be fake polarities: they are due to the intrinsic property of $G_{A}$ which is not a true helicity flux density. The simplest case is when a magnetic region is moving bodily (so no helicity is injected): $G_{A}$ has two strong polarities which can easily mask a real input of helicity, for example, by twisting motions (Sect. 4.1). Examples of these fake polarities associated with the global motion of a magnetic region can be found in all the above published maps of $G_{A}$; the clearest examples are in Nindos et al. (2003). The fake polarities induced by $G_{A}$ could represent significant, if not dominant, contributions to the observed positive and negative flux of magnetic helicity, maybe even masking the real helicity flux injection. The precision of the much lower net flux of helicity can be affected by this subtraction of close numbers (which, moreover, are affected by the periodicity used to compute $\boldsymbol{A}$ ).

In this study, the main objective was to solve the above problem by defining the flux of magnetic helicity per unit surface. In a first step, we define a new density, $G_{\theta}$ (Eq. (19)), by writing explicitly the vector potential $\boldsymbol{A}$ and rearranging the double summations over the magnetogram (Sect. 3.2). For an elementary magnetic region, $G_{\theta}$ sums up the rotation of all the other magnetic regions taking into account their magnetic flux. $G_{\theta}$ vanishes for one magnetic region moving uniformly, so, in this case, it solves the problem of $G_{A}$. However $G_{\theta}$ can still have weaker fake polarities when several magnetic regions are moving. For example when two magnetic regions are separating or approaching each other, two polarities of $G_{\theta}$ are present on each magnetic region (Sect. 4.2). They are usually weaker by more than one order of magnitude than those obtained with $G_{A}$ (Sects. 4.2 and 4.4) or even absent in some cases (e.g. Sect. 4.3). These results make $G_{\theta}$ much better suited to follow the injection of helicity in ARs, i.e. to detect the real flux density of magnetic helicity.

While $G_{\theta}$ will be of great practical use, it is still not the flux density of magnetic helicity. Indeed to define it one needs more information than the normal field component evolution (which is sufficient to compute $G_{\theta}$ ): we need the coronal connectivities for all the elementary flux tubes. This information enables us to define the helicity flux per elementary flux tube (Eq. (25)), a quantity which can be rewritten as a flux of magnetic helicity per unit surface $\left(G_{\Phi}\right.$, Eq. (28)). $G_{\Phi}$ is a field-weighted average of $G_{\theta}$ at both feet of the coronal connections, and it removes the remnant fake polarities associated to $G_{\theta}$ (Sects. 4.2 and 4.4). However some mixed polarities of $G_{\Phi}$ can be still present, e.g. when a coronal twisted field is moved by horizontal photospheric flows (Sect. 4.2.2). It is a clear warning that, even when using $G_{\Phi}$, the presence of both signs in the helicity flux density should be analyzed with care (e.g. it does not necessarily mean cancellation of magnetic helicity due to magnetic reconnection).

The present results are directly applicable to observations. They are independent of the method used to get the photospheric velocities. When local correlation tracking and/or the induction equation are used, one can deduced only the total flux density, but if plasma motions are available the flux density can be derived separately for the shear (horizontal motions) and advection (emergence) terms. While $G_{\Phi}$ provides the true helicity flux density, its practical use is presently limited by our ability to define the coronal linkage for all the magnetic polarities. This situation will be well improved in a near future with the high resolution and multi-wavelength observations of Solar-B (Shimizu 2002), as well as the development of numerical techniques to compute the coronal field from photospheric data. So presently $G_{\theta}$ is of most use, but it will be replaced by $G_{\Phi}$ later on. This will bring another step in detecting weaker input of helicity flux density (which when summed over an AR and time can bring a significant amount of magnetic helicity). It will also permit us to track the emergence of truly opposite magnetic polarity to the dominant one in an AR, a case which could be important for flaring (Kusano et al. 2004).

Acknowledgements. We thank the referee for her/his helpful comments.

\section{References}

Berger, M. A. 1984, Geophys. Astrophys. Fluid Dyn., 30, 79 Berger, M. A., \& Field, G. B. 1984, J. Fluid. Mech., 147, 133 Brown, D. S., Nightingale, R. W., Alexander, D., et al. 2003, Sol. Phys., 216, 79 
Chae, J. 2001, ApJ, 560, L95

Chae, J., Moon, Y., \& Park, Y. 2004, Sol. Phys., 223, 39

Chae, J., Wang, H., Qiu, J., et al. 2001, ApJ, 560, 476

Démoulin, P., \& Berger, M. A. 2003, Sol. Phys., 215, 203

Démoulin, P., Bagalá, L. G., Mandrini, C. H., Hénoux, J. C., \& Rovira, M. G. 1997, A\&A, 325, 305

Démoulin, P., Mandrini, C. H., van Driel-Gesztelyi, L., et al. 2002, A\&A, 382, 650

Emonet, T., \& Moreno-Insertis, F. 1998, ApJ, 492, 804

Finn, J. H., \& Antonsen, T. M. 1985, Comm. Plasma Phys. Contr. Fus., 9,111

Gibson, S., Fan, Y., Mandrini, C. H., Fisher, G., \& Démoulin, P. 2004, ApJ, 617, 600

Green, L. M., López fuentes, M. C., Mandrini, C. H., et al. 2002, Sol. Phys., 208, 43

Kusano, K., Maeshiro, T., Yokoyama, T., \& Sakurai, T. 2002, ApJ, 577,501
Kusano, K., Maeshiro, T., Yokoyama, T., \& Sakurai, T. 2004, ApJ, 610,537

Kusano, K., Maeshiro, T., Yokoyama, T., \& Sakurai, T. 2005, in The Solar-B Mission and the Forefront of Solar Physics, ed. T. Sakurai, \& T. Skii (San Francisco: ASP), ASP Conf. Ser., 325, in press

Leka, K. D., Canfield, R. C., McClymont, A. N., \& van Driel-Gesztelyi, L. 1996, ApJ, 462, 547

Longcope, D. W. 2004, ApJ, 612, 1181

Moon, Y.-J., Chae, J., Choe, G. S., et al. 2002a, ApJ, 574, 1066

Moon, Y.-J., Chae, J., Wang, H., Choe, G. S., \& Park, Y. D. 2002b, ApJ, 580, 528

Nindos, A., \& Zhang, H. 2002, ApJ, 573, L133

Nindos, A., Zhang, J., \& Zhang, H. 2003, ApJ, 594, 1033

Shimizu, T. 2002, Adv. Space Res., 29, 2009

Welsch, B. T., Fisher, G. H., Abbett, W. P., \& Regnier, S. 2004, ApJ, 610,1148

Welsch, B. T., \& Longcope, D. W. 2003, ApJ, 588, 620 
E. Pariat et al.: Photospheric flux density of magnetic helicity, Online Material p 1

\section{Online Material}




\section{Appendix A: Computation of $G_{\theta}$ for a polarity rotating around another one}

We complement in this appendix Sect. 4.3. The velocity field at a point $M\left(\in P_{-}\right)$given by Eq. (42) can be decomposed in two different movements:

$$
\boldsymbol{u}=\boldsymbol{\Omega} \times \boldsymbol{O}_{+} \boldsymbol{O}_{-}+\boldsymbol{\Omega} \times \boldsymbol{O}_{-} \boldsymbol{M} \text { for } M \in P_{-} .
$$

The first term in the right hand part of the equation corresponds to a rigid rotation of $P_{-}$around $P_{+}$and the second term to the rigid rotation of $P_{-}$around its centre.

\section{A.1. Computation of $\mathrm{G}_{\theta}$ in $P_{-}$}

The rotation rate between $M$ and another point $M^{\prime}$ also belonging to $P_{-}$is then simply deduced from Eq. (17):

$$
\begin{aligned}
\frac{\mathrm{d} \theta\left(\boldsymbol{M}^{\prime} \boldsymbol{M}\right)}{\mathrm{d} t} & =\frac{\left(\boldsymbol{M}^{\prime} \boldsymbol{M} \times\left(\boldsymbol{\Omega} \times \boldsymbol{O}_{-} \boldsymbol{M}-\boldsymbol{\Omega} \times \boldsymbol{O}_{-} \boldsymbol{M}^{\prime}\right)\right)_{n}}{\left|M M^{\prime}\right|^{2}} \\
& =\Omega \quad \text { for }\left(M, M^{\prime}\right) \in\left(P_{-}\right)^{2}
\end{aligned}
$$

This simply corresponds to the rigid rotation of $P_{-}$around itself - the twist.

The writhe is given when $M^{\prime}$ belongs to $P_{+}$; the rotation rate is then a bit more complex:

$\frac{\mathrm{d} \theta\left(\boldsymbol{M}^{\prime} \boldsymbol{M}\right)}{\mathrm{d} t}=\Omega \frac{\boldsymbol{M}^{\prime} \boldsymbol{M} \cdot \boldsymbol{O}_{+} \boldsymbol{M}}{\left|\boldsymbol{M} M^{\prime}\right|^{2}}$

with $M \in P_{-}$and $M^{\prime} \in P_{+}$.

Thus, using Eqs. (A.2), (A.4) in Eq. (19), $G_{\theta}(\boldsymbol{x})$ in $P_{-}$is equal to:

$G_{\theta}(\boldsymbol{x})=\frac{\Omega B_{0}^{2}}{2 \pi}\left(\int_{M^{\prime} \in P_{+}} \frac{\boldsymbol{O}_{+} \boldsymbol{M} \cdot \boldsymbol{M}^{\prime} \boldsymbol{M}}{\left|M M^{\prime}\right|^{2}} \mathrm{~d} \mathcal{S}^{\prime} \int_{M^{\prime} \in P_{-}} \mathrm{d} \mathcal{S}^{\prime}\right)$

$$
\text { for } M \in P_{-} \text {. }
$$

The first term in the right hand part of Eq. (A.4) can be computed by analogy to the $2 \mathrm{D}$ electric field created by a uniform distribution of charge:

$\int_{M^{\prime} \in P_{+}} \frac{\boldsymbol{M}^{\prime} \boldsymbol{M}}{\left|M M^{\prime}\right|^{2}} \mathrm{~d} \mathcal{S}^{\prime}=\pi R^{2} \frac{\boldsymbol{O}_{+} \boldsymbol{M}}{\left|O_{+} M\right|^{2}}$, and so,

$G_{\theta}(\boldsymbol{x})=0 \quad$ for $M \in P_{-}$.

Thus there is no injection of helicity in the moving polarity. The injection of negative helicity due to the twist is exactly compensated by the positive writhe helicity due to the relative motion of $P_{+}$around $P_{-}$.

\section{A.2. Computation of $G_{\theta}$ in $P_{+}$}

In the case where $M$ is in the polarity $P_{+}$, which is not moving, the rotation rate is only non-null for the points $M^{\prime}$ located in the opposite polarity $P_{-}$, and can be expressed as:

$\frac{\mathrm{d} \theta\left(\boldsymbol{M}^{\prime} \boldsymbol{M}\right)}{\mathrm{d} t}=\Omega\left(1+\frac{\boldsymbol{M} \boldsymbol{M}^{\prime} \cdot \boldsymbol{O}_{+} \boldsymbol{M}}{\left|\boldsymbol{M} M^{\prime}\right|^{2}}\right)$

with $M \in P_{+}$and $M^{\prime} \in P_{-}$.

where a constant rotation appears. One then obtains for $G_{\theta}$ :

$$
\begin{aligned}
G_{\theta}(\boldsymbol{x})= & \frac{\Omega B_{0}^{2}}{2 \pi}\left(\boldsymbol{O}_{+} \boldsymbol{M} \cdot \int_{M^{\prime} \in P_{-}} \frac{\boldsymbol{M} \boldsymbol{M}^{\prime}}{\left|M M^{\prime}\right|^{2}} \mathrm{~d} \mathcal{S}^{\prime}\right. \\
& \left.+\int_{M^{\prime} \in P_{-}} \mathrm{d} \mathcal{S}^{\prime}\right) \quad \text { for } M \in P_{+} .
\end{aligned}
$$

The first term of the right hand part of this equation can be computed similarly as in Eq. (A.5):

$$
\begin{aligned}
G_{\theta}(\boldsymbol{x}) & =\frac{\Omega R^{2} B_{0}^{2}}{2}\left(1+\frac{\boldsymbol{O}_{+} \boldsymbol{M} \cdot \boldsymbol{M} \boldsymbol{O}_{-}}{\left|O_{-} M\right|^{2}}\right) \\
& =\frac{\Omega R^{2} B_{0}^{2}}{2} \frac{\boldsymbol{O}_{-} \boldsymbol{M} \cdot \boldsymbol{O}_{-} \boldsymbol{O}_{+}}{\left|O_{-} M\right|^{2}} \text { for } M \in P_{+},
\end{aligned}
$$

which demonstrates Eq. (45). Since one also has:

$$
\int_{M \in P_{+}} \frac{\boldsymbol{O}_{-} \boldsymbol{M}}{\left|O_{-} M\right|^{2}} \mathrm{~d} \mathcal{S}^{\prime}=\pi R^{2} \frac{\boldsymbol{O}_{-} \boldsymbol{O}_{+}}{\left|O_{-} O+\right|^{2}},
$$

it is possible to compute the total helicity flux injection:

$\frac{\mathrm{d} H}{\mathrm{~d} t}=\frac{\pi \Omega B_{0}^{2} R^{4}}{2}$,

a result identical, but more general, than the point-like result of Eq. (43). 